\title{
A QUÍMICA SUPRAMOLECULAR DE COMPLEXOS PIRAZÓLICOS
}

\author{
Adelino Vieira de Godoy Netto*, Regina Célia Galvão Frem e Antonio Eduardo Mauro \\ Departamento de Química Geral e Inorgânica, Instituto de Química, Universidade Estadual Paulista, CP 355, 14801-970 \\ Araraquara - SP, Brasil
}

Recebido em 29/6/07; aceito em 20/9/07; publicado na web em 9/4/08

SUPRAMOLECULAR CHEMISTRY OF PYRAZOLYL COMPLEXES. The purpose of this review is to describe the progress in the supramolecular chemistry of the pyrazolyl-based metal complexes. The text is written under the structural point of view, emphasizing the role of the covalent and non-covalent interactions in the rational construction of super and supramolecules.

Keywords: pyrazolyl transition metal compounds; covalent and non-covalent interactions; supramolecular architectures.

\section{INTRODUÇÃO}

Azóis são heterocíclicos aromáticos contendo um átomo de nitrogênio e um outro heteroátomo dispostos na posição-1,2 de um anel de cinco membros. Os pirazóis pertencem à família dos $1,2-$ azóis, juntamente com os isotiazóis e os isoxazóis, ${ }^{1-3}$ cujas fórmulas estruturais estão ilustradas na Figura 1.

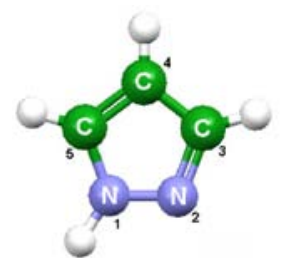

PIRAZOL

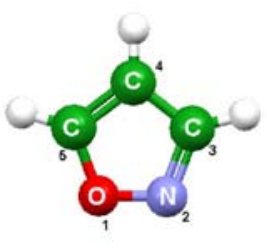

ISOXAZOL

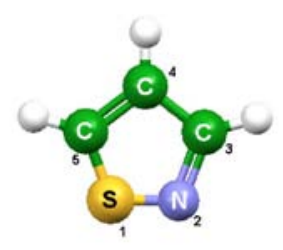

ISOTIAZOL
Figura 1. Fórmulas estruturais dos 1,2-azóis

Embora complexos pirazólicos sejam conhecidos há bastante tempo, como o $[\mathrm{Ag}(\mu-\mathrm{Pz})]_{\mathrm{n}}$, descrito por Buchner ${ }^{3,4}$ no final do século XIX, somente a partir de 1972 o interesse sobre o tema foi renovado com a publicação de um trabalho de Trofimenko. ${ }^{4} \mathrm{Ou}-$ tros trabalhos de revisão na área foram publicados por Trofimenko, ${ }^{5}$ Sadimenko et al. ${ }^{6}$ e Ardizzoia et al. ${ }^{7}$

Os pirazóis possuem uma química de coordenação rica pela versatilidade de modos de coordenação que podem apresentar. Assim sendo, esses compostos podem atuar como ligantes neutros monodentados (a), aniônicos monodentados (b), aniônicos exobidentados (c), aniônicos endobidentados (d) ou como ligante penta-hapto, $\eta^{5}$ (e), conforme ilustra a Figura 2.

O modo de coordenação neutro monodentado do pirazol é evidenciado no trabalho de Netto et al. ${ }^{8}$ no qual estão descritas a obtenção e a caracterização estrutural do complexo trans$\left[\mathrm{Pd}(\mathrm{SCN})_{2}(\mathrm{HPz})_{2}\right]$ (Figura 3).

A possibilidade de desprotonação do nitrogênio pirrólico dos pirazóis leva à formação do ânion pirazolato. Este ligante aniônico pode atuar no modo de coordenação monodentado (simetria $C_{s}$ ), bidentado (simetria $C_{2 v}$ ), ou $\eta^{5}$ de maneira similar aos metalocenos.

Fackler et al. ${ }^{9}$ relataram a preparação do complexo cis$\left[\mathrm{PtCl}(\mathrm{dfPz})\left(\mathrm{PPh}_{3}\right)_{2}\right]$ no qual o ligante 3,5-difenilpirazolato encon-

*e-mail: adelino@iq.unesp.br

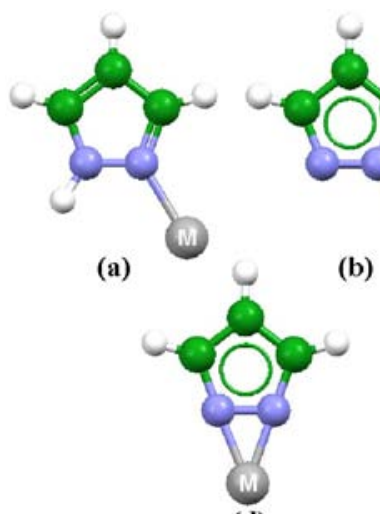

(d)

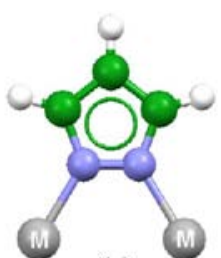

(c)
Figura 2. Modos de coordenação dos ligantes pirazólicos $(M=$ metal $)$

tra-se coordenado no modo aniônico monodentado (Figura 4).

Quanto ao modo bidentado dos pirazolatos, duas possibilidades de coordenação podem ocorrer. Quando os dois átomos de nitrogênio do grupo pirazolato se ligam simultaneamente ao mesmo centro metálico, o modo de coordenação é denominado endobidentado e foi verificado no trabalho de Yélamos et al. ${ }^{10}$ que relataram a determinação estrutural do complexo $\left[\mathrm{HfCl}(\mathrm{dbPz})_{3}\right]$ (Figura 5).

A segunda possibilidade de coordenação envolve o modo exobidentado, no qual o ânion pirazolato atua como ligante ponte entre dois centros metálicos, como relatado por Pettinari et al. ${ }^{11}$ para o complexo [(dppf)Pt $\left.(\mu-\mathrm{Pz})_{2} \mathrm{CdI}_{2}\right]$ (Figura 6).

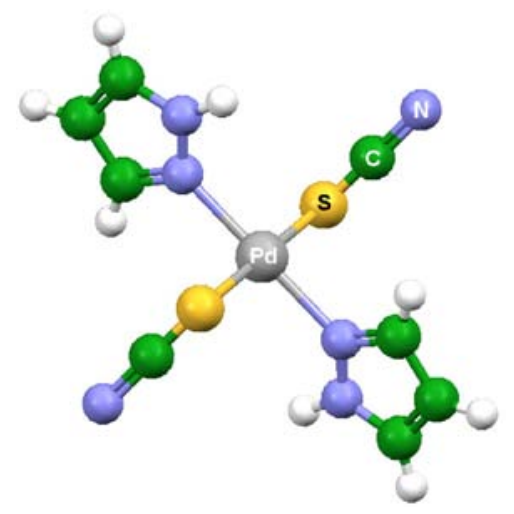

Figura 3. Fórmula estrutural do complexo trans-[Pd(SCN $\left.)_{2}(\mathrm{HPz})_{2}\right]$ 


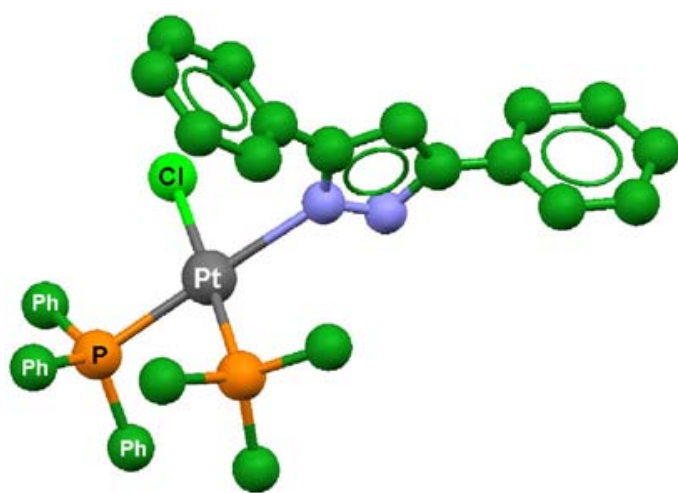

Figura 4. Fórmula estrutural do composto cis-[PtCl(dfPz $\left.)\left(\mathrm{PPh}_{3}\right)_{2}\right]$

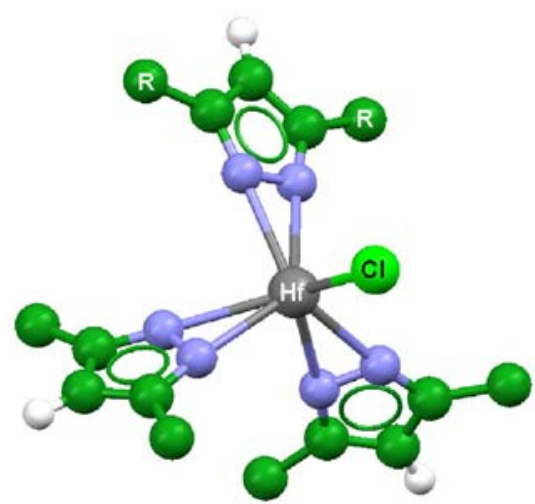

Figura 5. Fórmula estrutural de $\left[\mathrm{HfCl}(\mathrm{dbPz})_{3}\right]\left(R={ }^{\mathrm{t}} \mathrm{Bu}\right)$

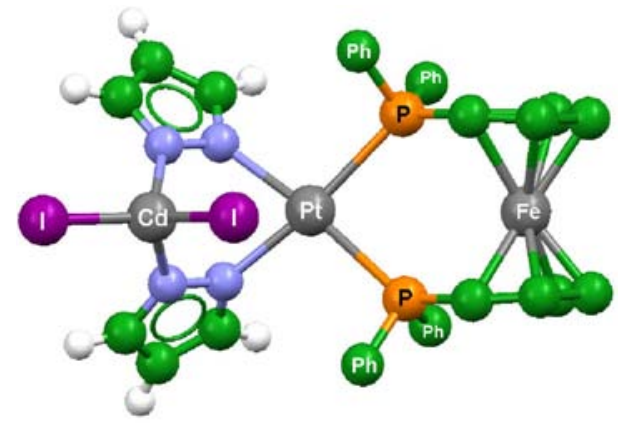

Figura 6. Fórmula estrutural de [(dppf)Pt( $\left.\mu-P z)_{2} C_{2} I_{2}\right]$

No trabalho de Perera et al. ${ }^{12}$ é descrito o composto $\left[\mathrm{Ru}\left(\eta^{5}\right.\right.$ $\left.\mathrm{dmPz})\left(\eta^{5}-\mathrm{Cp}\right)\right]$ (Figura 7) no qual o 3,5-dimetilpirazolato $(\mathrm{dmPz})$ encontra-se coordenado no modo penta-hapto $\left(\eta^{5}\right)$, de maneira semelhante ao ânion ciclopentadienil (Cp).

\section{Aplicações}

Os complexos pirazólicos suscitam cada vez mais atenção não somente pelas suas diversidades estruturais, mas também por suas potencialidades de aplicação em catálise homogênea, como materiais moleculares e na química inorgânica medicinal. Dentro dessa perspectiva, espécies binucleares de $\mathrm{Rh}$ (II) são utilizadas como catalisadores em reações de hidroformilação de olefinas ${ }^{13}$ e complexos pirazólicos de $\mathrm{Ni}(\mathrm{II})$ e $\mathrm{Pd}(\mathrm{II})$ catalisam a polimerização do etileno. ${ }^{14}$ Outra característica importante desta classe de compostos reside em suas propriedades líquido-cristalinas. Sendo assim, é relatado na literatura que compostos mononucleares de $\mathrm{Pd}(\mathrm{II}) \mathrm{e}$ trímeros de $\mathrm{Au}(\mathrm{I})$, contendo ligantes pirazólicos, são capazes de formar mesofases calamíticas ${ }^{15}$ e discóticas, ${ }^{16}$ respectivamente. No que concerne a atividade biológica de complexos pirazólicos, compostos de $\mathrm{Au}(\mathrm{I})$ têm demonstrado atividade anti-microbiana seletiva frente a determinadas bactérias e leveduras. ${ }^{17}$ Muito esforço é dedicado ao desenvolvimento de novas metalodrogas quimioterápicas para o tratamento do câncer. Nesse contexto, Komeda et $a l .{ }^{18}$ verificaram que o complexo binuclear $\left[\left(\mathrm{NH}_{3}\right)_{2} \mathrm{Pt}(\mu-\mathrm{Pz})(\mu-\right.$ $\left.\mathrm{OH}) \mathrm{Pt}\left(\mathrm{NH}_{3}\right)_{2}\right]\left(\mathrm{NO}_{3}\right)_{2}$ é muito mais efetivo e citotóxico que a cisplatina frente a algumas linhagens cancerosas. Complexos pirazólicos de paládio(II) também mostraram potente atividade citotóxica $^{19}$ frente a células leucêmicas P388.

\section{A QUÍMICA SUPRAMOLECULAR DE COMPLEXOS PIRAZÓLICOS}

A Química Supramolecular, definida como a "química além da molécula", está relacionada a entidades organizadas de alta complexidade, resultantes da associação de duas ou mais espécies químicas, moléculas e/ou íons, unidas por ligações metal-ligante e/ou interações intermoleculares não-covalentes, tais como interações eletrostáticas, ligações de hidrogênio, forças de van der Waals, entre outras. ${ }^{20}$ Neste contexto, os próprios ligantes pirazólicos livres podem se auto-organizar no estado sólido, mediante ligações de hidrogênio, formando espécies supramoleculares do tipo dímeros, trímeros, tetrâmeros e cadeias infinitas conhecidas como catâmeros. ${ }^{21}$ A título de ilustração, a Figura 8 mostra a representação do trímero supramolecular.

Por outro lado, apesar da química de coordenação dos pirazóis ser bem investigada, o emprego destes ligantes na construção de espécies metalossupramoleculares, a partir de reações de auto-montagem envolvendo metais de transição de diferentes geometrias, encontra-se ainda em desenvolvimento. Sob esse ponto de vista, o objetivo desta parte da revisão é apresentar o estado-da-arte da química supramolecular dos pirazóis, com ênfase nos aspectos estruturais. Cabe salientar que foi realizada uma compilação detalhada dos prin-

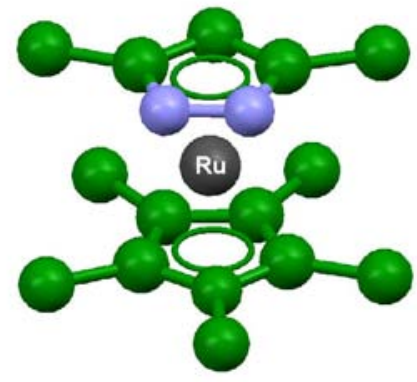

Figura 7. Fórmula estrutural do complexo $\left[R u\left(\eta^{5}-d m P z\right)\left(\eta^{5}-C p\right)\right]$

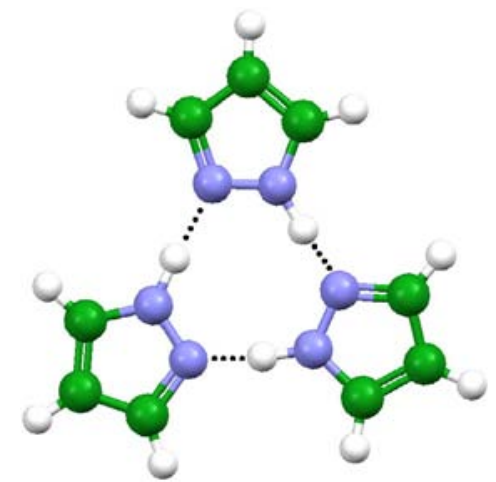

Figura 8. Trímero supramolecular de pirazóis 
cipais trabalhos realizados nos últimos anos pela comunidade científica, excetuando-se aqueles envolvendo pirazóis funcionalizados, o que tornaria esse artigo excessivamente extenso.

O fato dos pirazóis apresentarem dois átomos de nitrogênio quimicamente diferentes, $\mathrm{N}$-pirrólico e $\mathrm{N}$-piridínico, torna-os blocos de montagens promissores na construção de diversas estruturas inorgânicas. Deste modo, podem ser construídas desde arquiteturas discretas e poliméricas mediante formação de ligações coordenadas até espécies supramoleculares auto-organizadas por ligações de hidrogênio e outras interações não covalentes.

\section{Arquiteturas discretas}

Os triângulos moleculares constituem uma das espécies supramoleculares pirazólicas mais estudadas, ${ }^{22-26}$ que são automontadas por ânions pirazolatos (unidade angular) e íons metálicos de geometria de coordenação linear.

Vários autores têm aplicado esta metodologia de síntese, com sucesso, na obtenção de trímeros pirazólicos (Figura 9), variando a natureza dos grupos substituintes do anel pirazolato bem como o centro metálico $\left(\mathrm{Cu}^{+}, \mathrm{Ag}^{+}, \mathrm{Au}^{+}\right)$.

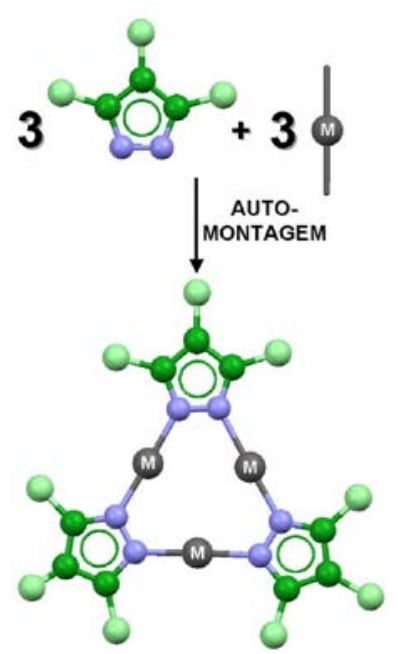

Figura 9. Representação esquemática da auto-montagem de trímeros moleculares a partir de grupos pirazolatos e ions metálicos de geometria de coordenação linear $(M=$ metal $)$

Ainda que em número substancialmente reduzido, encontramse na literatura alguns trabalhos envolvendo a construção de triângulos pirazólicos contendo centros metálicos de geometria quadrado planar. Um exemplo envolve o íon $\mathrm{Pt}(\mathrm{II})$ no composto $[\mathrm{Pt}(\mu-$ $\left.\mathrm{Pz})_{2}\right]_{3}$ relatado por Burger et al., ${ }^{26}$ cuja representação estrutural encontra-se na Figura 10

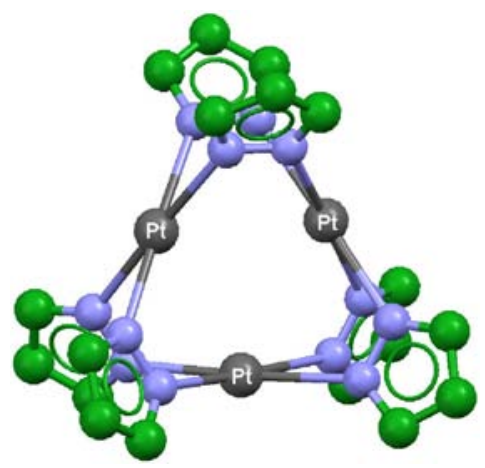

Figura 10. Fórmula estrutural do trímero $\left[\operatorname{Pt}(\mu-P z)_{2}\right]_{3}$
A Tabela 1 contempla os trímeros pirazólicos descritos na literatura, especificando a natureza do centro metálico e o tipo de ligante empregado.

Além dos trímeros, espécies de nuclearidades superiores são obtidas dependendo do tipo de pirazol empregado, da geometria de coordenação do centro metálico e das condições de síntese. ${ }^{7}$ No que se refere a tetrâmeros moleculares contendo pirazóis, são conhecidas apenas algumas espécies com íons de geometria de coordenação linear $\mathrm{Cu}(\mathrm{I}), \mathrm{Ag}(\mathrm{I})$ e $\mathrm{Au}(\mathrm{I}) .{ }^{27} \mathrm{O}$ tetrâmero $[\mathrm{Cu}(\mu \text {-dfPz })]_{4}$ obtido por Ardizzoia et al. ${ }^{27}$ serve como exemplo dessa classe de compostos tetranucleares e está representado na Figura 11. A Tabela 2 apresenta uma compilação dos trabalhos envolvendo tetrâmeros pirazólicos.

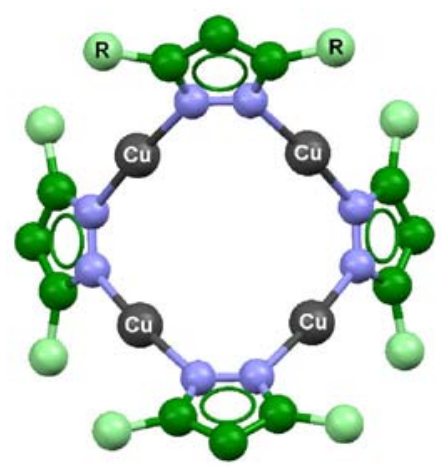

Figura 11. Fórmula estrutural do tetrâmero $[\mathrm{Cu}(\mu-d f P z)]_{4}(R=P h)$

O próximo composto da série envolve o hexâmero $[\mathrm{Au}(\mu$ dfPz) $]_{6}$, sintetizado por Murray et al., ${ }^{23,28}$ e trata-se do único exemplo de uma espécie inorgânica homoléptica hexanuclear contendo pirazolato.

No que se refere a polígonos de nuclearidade mais alta, metalociclos heterolépticos neutros de fórmula geral $[\mathrm{Cu}(\mu-\mathrm{OH})(\mu-$ $\mathrm{Pz})]_{\mathrm{n}}(\mathrm{n}=6,8,9,12,14)$ foram descritos por Raptis et al. ${ }^{29}$ (Figura 12). Esses anéis consistem de centros de $\mathrm{Cu}(\mathrm{II})$ quadrado-planares distorcidos conectados por ligantes pirazolatos orientados para a superfície externa e grupos $\mathrm{OH}^{-}$direcionados para a cavidade interna da estrutura.

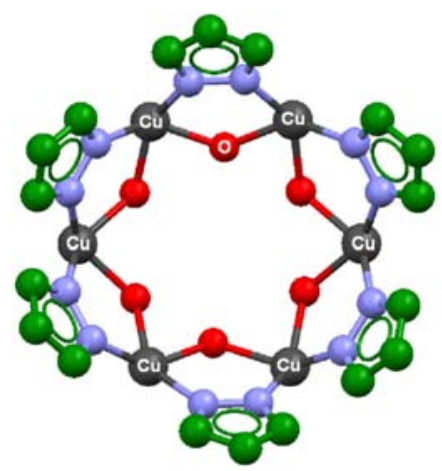

Figura 12. Representação estrutural do metalociclo $[\mathrm{Cu}(\mu-\mathrm{OH})(\mu-\mathrm{Pz})]_{6}$

No trabalho de Ardizzoia et al. ${ }^{30}$ foi descrita a preparação da espécie octanuclear $[\mathrm{Cu}(\mu-\mathrm{OH})(\mu-\mathrm{dmPz})]_{8}$, na qual os grupos $\mathrm{OH}^{-}$ e dimetilpirazolato encontram-se alternados na estrutura cíclica, em função da presença dos substituintes nas posições 3 e 5 do ligante pirazólico. O diâmetro estimado da cavidade é de aproximadamente $6 \AA$ e em seu empacotamento cristalino há a formação de canais infinitos pelo empilhamento das unidades octaméricas. 
Tabela 1.Triângulos moleculares pirazólicos de fórmula geral $\left[\mathrm{M}(\mu-\mathrm{L})_{1-2}\right]_{3}$

\begin{tabular}{|c|c|c|c|c|c|}
\hline M & $\mathrm{R}_{3}$ & $\mathrm{R}_{4}$ & $\mathrm{R}_{5}$ & $\mathrm{~L}$ & Ref. \\
\hline $\mathrm{Cu}$ & $\mathrm{CH}_{3}$ & $\mathrm{NO}_{2}$ & $\mathrm{CH}_{3}$ & $\mathrm{dmnPz}$ & 22 \\
\hline $\mathrm{Cu}$ & $\mathrm{CH}_{3}^{3}$ & $\mathrm{H}^{2}$ & $\mathrm{CH}_{3}^{3}$ & $\mathrm{dmPz}$ & 22 \\
\hline $\mathrm{Cu}$ & $\mathrm{CF}_{3}$ & $\mathrm{H}$ & $\mathrm{H}^{3}$ & $\mathrm{fmPz}$ & 22 \\
\hline $\mathrm{Cu}$ & $\mathrm{CF}_{3}^{3}$ & $\mathrm{H}$ & $\mathrm{CH}_{3}$ & $\mathrm{fmmPz}$ & 22 \\
\hline $\mathrm{Cu}$ & $\mathrm{CF}_{3}^{3}$ & $\mathrm{H}$ & $\mathrm{C}_{6} \mathrm{H}_{5}$ & $\mathrm{fmfPz}$ & 22 \\
\hline $\mathrm{Cu}$ & $\mathrm{C}_{6} \mathrm{H}_{5}$ & $\mathrm{H}$ & $\mathrm{C}_{6} \mathrm{H}_{5}$ & $\mathrm{dfPz}$ & 22 \\
\hline $\mathrm{Cu}$ & $\mathrm{C}_{3} \mathrm{H}_{7}$ & $\mathrm{H}$ & $\mathrm{C}_{3} \mathrm{H}_{7}$ & $\mathrm{dpPz}$ & 22 \\
\hline $\mathrm{Cu}$ & $\mathrm{CF}_{3}$ & $\mathrm{H}$ & $\mathrm{CF}_{3}$ & $\mathrm{dfmPz}$ & 22 \\
\hline $\mathrm{Ag}$ & $\mathrm{H}^{3}$ & $\mathrm{H}$ & $\mathrm{H}^{3}$ & $\mathrm{Pz}$ & 23 \\
\hline $\mathrm{Ag}$ & $\mathrm{C}_{6} \mathrm{H}_{5}$ & $\mathrm{H}$ & $\mathrm{C}_{6} \mathrm{H}_{5}$ & $\mathrm{dfPz}$ & 23 \\
\hline $\mathrm{Ag}$ & $\mathrm{C}_{3}^{6} \mathrm{H}_{7}^{3}$ & $\mathrm{H}$ & $\mathrm{C}_{3} \mathrm{H}_{7}^{3}$ & $\mathrm{dpPz}$ & 23 \\
\hline $\mathrm{Ag}$ & $\mathrm{C}_{3} \mathrm{H}_{7}$ & $\mathrm{Br}$ & $\mathrm{C}_{3} \mathrm{H}_{7}^{\prime}$ & $\mathrm{dpbPz}$ & 23 \\
\hline $\mathrm{Ag}$ & $\mathrm{C}_{3}^{3} \mathrm{H}_{7}^{\prime}$ & $\mathrm{NO}_{2}$ & $\mathrm{C}_{3}^{3} \mathrm{H}_{7}$ & $\mathrm{dpnPz}$ & 23 \\
\hline $\mathrm{Ag}$ & $\mathrm{CF}_{3}$ & $\mathrm{H}^{2}$ & $\mathrm{CF}_{3}$ & $\mathrm{dfmPz}$ & 22,23 \\
\hline $\mathrm{Ag}$ & $\mathrm{C}_{4} \mathrm{H}_{9}$ & $\mathrm{H}$ & $\mathrm{CF}_{3}^{3}$ & $\mathrm{bfmPz}$ & 23 \\
\hline $\mathrm{Ag}$ & $\mathrm{C}_{4}^{4} \mathrm{H}_{9}$ & $\mathrm{H}$ & $\mathrm{C}_{4} \mathrm{H}_{9}$ & $\mathrm{dbPz}$ & 23 \\
\hline $\mathrm{Ag}$ & $\mathrm{C}_{6}^{4} \mathrm{H}_{4} \mathrm{OR}$ & $\mathrm{H}$ & $\mathrm{H}^{4}$ & aoxfPz & 23 \\
\hline $\mathrm{Ag}$ & $\mathrm{C}_{6} \mathrm{H}_{4} \mathrm{OPh}$ & $\mathrm{H}$ & $\mathrm{H}$ & foxfPz & 23 \\
\hline $\mathrm{Ag}$ & $\mathrm{C}_{6}^{6} \mathrm{H}_{4}^{4} \mathrm{OR}$ & $\mathrm{H}$ & $\mathrm{C}_{6} \mathrm{H}_{4} \mathrm{OR}$ & daoxfPz & 23 \\
\hline $\mathrm{Ag}$ & $\mathrm{C}_{6} \mathrm{H}_{4}^{4} \mathrm{OPh}$ & $\mathrm{H}$ & $\mathrm{C}_{6} \mathrm{H}_{4}^{4} \mathrm{OPh}$ & dfozfPz & 23 \\
\hline $\mathrm{Au}$ & $\mathrm{CF}_{3}^{4}$ & $\mathrm{H}$ & $\mathrm{CF}_{3}^{4}$ & $\mathrm{dfmPz}$ & 22,24 \\
\hline $\mathrm{Au}$ & $\mathrm{CH}_{3}^{3}$ & $\mathrm{C}_{\mathrm{n}} \mathrm{H}_{2 \mathrm{n}+1}(\mathrm{n}=7-11)$ & $\mathrm{CH}_{3}^{3}$ & $\mathrm{dmaPz}$ & 24 \\
\hline $\mathrm{Au}$ & $\mathrm{C}_{8} \mathrm{H}_{17}^{3}$ & $\mathrm{H}^{\mathrm{n}}$ & $\mathrm{C}_{8} \mathrm{H}_{17}^{3}$ & $\mathrm{doPz}$ & 24 \\
\hline $\mathrm{Au}$ & $\mathrm{C}_{6} \mathrm{H}_{4}\left(\mathrm{OC}_{10} \mathrm{H}_{21}\right)_{2}$ & $\mathrm{H}$ & $\mathrm{C}_{6}^{8} \mathrm{H}_{4}\left(\mathrm{OC}_{10} \mathrm{H}_{21}\right)_{2}$ & ddoxfPz & 24 \\
\hline $\mathrm{Au}$ & $\mathrm{C}_{6}^{6} \mathrm{H}_{4}\left(\mathrm{OC}_{10} \mathrm{H}_{21}\right)_{2}^{2}$ & $\mathrm{H}$ & $\mathrm{C}_{6}^{6} \mathrm{H}_{4}\left(\mathrm{OC}_{10}^{10} \mathrm{H}_{21}\right)_{3}$ & dtdoxfPz & 24 \\
\hline $\mathrm{Au}$ & $\mathrm{CH}_{3}$ & $\mathrm{C}_{6} \mathrm{H}_{3} \mathrm{CH}_{2}\left(\mathrm{OC}_{18} \mathrm{H}_{37}\right)_{2}$ & $\mathrm{CH}_{3}{ }^{4} \quad 10 \quad 213$ & dmodoxbPz & 24 \\
\hline $\mathrm{Au}$ & $\mathrm{H}^{3}$ & $\mathrm{H}^{6}-2 \mathrm{l}-18-37 / 2$ & $\mathrm{H}^{3}$ & $\mathrm{Pz}$ & 24 \\
\hline $\mathrm{Au}$ & $\mathrm{H}$ & $\mathrm{CH}_{3}$ & $\mathrm{H}$ & $4-\mathrm{mPz}$ & 24 \\
\hline $\mathrm{Au}$ & $\mathrm{CH}_{3}$ & $\mathrm{H}^{3}$ & $\mathrm{C}_{6} \mathrm{H}_{5}$ & $\mathrm{mfPz}$ & 24 \\
\hline $\mathrm{Au}$ & $\mathrm{CH}_{3}^{3}$ & $\mathrm{H}$ & $\mathrm{CH}_{3}^{3}$ & $\mathrm{dmPz}$ & 24 \\
\hline $\mathrm{Au}$ & $\mathrm{C}_{6} \mathrm{H}_{5}^{3}$ & $\mathrm{H}$ & $\mathrm{C}_{6} \mathrm{H}_{5}$ & $\mathrm{dfPz}$ & 23 \\
\hline $\mathrm{Au}$ & $\mathrm{C}_{6}^{6} \mathrm{H}_{4}^{3} \mathrm{OR}$ & $\mathrm{H}$ & $\mathrm{H}^{3}$ & aoxfPz & 23 \\
\hline $\mathrm{Au}$ & $\mathrm{C}_{6} \mathrm{H}_{4} \mathrm{OPh}$ & $\mathrm{H}$ & $\mathrm{H}$ & foxfPz & 23 \\
\hline $\mathrm{Au}$ & $\mathrm{C}_{6}^{6} \mathrm{H}_{4}^{4} \mathrm{OR}$ & $\mathrm{H}$ & $\mathrm{C}_{6} \mathrm{H}_{4} \mathrm{OR}$ & daoxfPz & 23 \\
\hline $\mathrm{Au}$ & $\mathrm{C}_{6} \mathrm{H}_{4} \mathrm{OPh}$ & $\mathrm{H}$ & $\mathrm{C}_{6} \mathrm{H}_{4} \mathrm{OPh}$ & dfozfPz & 23 \\
\hline $\mathrm{Pd}$ & $\mathrm{C}_{6}^{6} \mathrm{H}_{5}^{4}$ & $\mathrm{H}$ & $\mathrm{H}^{64}$ & $\mathrm{fPz}$ & 25 \\
\hline $\mathrm{Pd}$ & $\mathrm{H}$ & $\mathrm{H}$ & $\mathrm{H}$ & $\mathrm{Pz}$ & 25 \\
\hline $\mathrm{Pd}$ & $\mathrm{H}$ & $\mathrm{CH}_{3}$ & $\mathrm{H}$ & 4-mPz & 25 \\
\hline $\mathrm{Pt}$ & $\mathrm{H}$ & $\mathrm{H}$ & $\mathrm{H}$ & $\mathrm{Pz}$ & 26 \\
\hline
\end{tabular}

\section{Polímeros de coordenação}

Segundo Janiak, ${ }^{31}$ polímeros de coordenação podem ser definidos como compostos de coordenação que se estendem infinitamente em 1, 2 ou 3 dimensões via ligações metal-ligante. Nesse contexto, a capacidade de grupos pirazolatos de atuarem como ligantes exobidentados entre dois centros metálicos adjacentes possibilita a construção de polímeros de coordenação. Conforme mencionado, a espécie polimérica $[\mathrm{Ag}(\mu-\mathrm{Pz})]_{\mathrm{n}}$ foi o primeiro composto pirazólico relatado na literatura por Buchner, ${ }^{3,4}$ em 1889. Entretanto, as primeiras investigações sistemáticas de polímeros de coordenação pirazólicos iniciaram-se apenas em 1973, com os trabalhos de Singh et al. ${ }^{32}$ sobre complexos polinucleares de fórmula geral $\left[\mathrm{M}(\mu-\mathrm{dmPz})_{2}\right]_{\mathrm{n}}\{\mathrm{M}=\mathrm{Co}(\mathrm{II}), \mathrm{Ni}(\mathrm{II}), \mathrm{Cu}(\mathrm{II})\}$, e intensificaramse alguns anos mais tarde com os estudos espectroscópicos de Vos e Groeneveld. ${ }^{33-37}$ A Tabela 3 apresenta um resumo dos trabalhos da literatura sobre polímeros pirazólicos de coordenação, especificando o tipo de pirazolato e o metal de transição.

A insolubilidade é o principal fator que dificulta a caracteriza-
Tabela 2. Tetrâmeros moleculares pirazólicos de fórmula geral $[\mathrm{M}(\mu-\mathrm{L})]_{4}$

\begin{tabular}{lccccc}
\hline $\mathrm{M}$ & $\mathrm{R}_{3}$ & $\mathrm{R}_{4}$ & $\mathrm{R}_{5}$ & $\mathrm{~L}$ & Ref. \\
\hline $\mathrm{Cu}$ & $\mathrm{C}_{6} \mathrm{H}_{5}$ & $\mathrm{H}$ & $\mathrm{C}_{6} \mathrm{H}_{5}$ & $\mathrm{dfPz}$ & 27 \\
$\mathrm{Cu}$ & $\mathrm{C}_{4} \mathrm{H}_{9}$ & $\mathrm{H}$ & $\mathrm{C}_{4} \mathrm{H}_{9}$ & $\mathrm{dbPz}$ & 22,27 \\
$\mathrm{Cu}$ & $\mathrm{COOC}_{4} \mathrm{H}_{9}$ & $\mathrm{H}$ & $\mathrm{COOC}_{4} \mathrm{H}_{9}$ & $\mathrm{dcbPz}$ & 27 \\
$\mathrm{Cu}$ & $\mathrm{C}_{4} \mathrm{H}_{9}$ & $\mathrm{H}$ & $\mathrm{C}_{3} \mathrm{H}_{7}$ & $\mathrm{bpPz}$ & 22 \\
$\mathrm{Ag}$ & $\mathrm{C}_{4} \mathrm{H}_{9}$ & $\mathrm{H}$ & $\mathrm{C}_{4} \mathrm{H}_{9}$ & $\mathrm{dbPz}$ & 27 \\
$\mathrm{Au}$ & $\mathrm{C}_{4} \mathrm{H}_{9}$ & $\mathrm{H}$ & $\mathrm{C}_{4} \mathrm{H}_{9}$ & $\mathrm{dbPz}$ & 27 \\
\hline
\end{tabular}

ção dos polímeros de coordenação pelas técnicas convencionais. Além disso, muitos deles são obtidos na forma de pós amorfos ou sólidos microcristalinos, inadequados para o estudo cristalográfico por difração de raios-X de monocristal. Mesmo diante dessas dificuldades, Ehlert et al. determinaram, pela primeira vez, a estrutura molecular e cristalina dos polímeros $\left[\mathrm{Cu}(\mu-4-\mathrm{XPz})_{2}\right]_{\mathrm{n}}(\mathrm{X}=\mathrm{Cl}, \mathrm{Br}$, $\left.\mathrm{CH}_{3}, \mathrm{H}\right)$ via difração de raios- $\mathrm{X}$ de monocristal. ${ }^{38,39} \mathrm{O}$ composto 
Tabela 3. Polímeros pirazólicos de coordenação homolépticos de fórmula geral $\left[\mathrm{M}(\mu-\mathrm{L})_{1-3}\right]_{\mathrm{n}}$

\begin{tabular}{|c|c|c|c|c|c|}
\hline M & $\mathrm{R}_{3}$ & $\mathrm{R}_{4}$ & $\mathrm{R}_{5}$ & $\mathrm{~L}$ & Ref. \\
\hline $\mathrm{Ag}^{+}$ & $\mathrm{H}$ & $\mathrm{H}$ & $\mathrm{H}$ & $\mathrm{Pz}$ & 3,4 \\
\hline $\mathrm{Co}^{2+}, \mathrm{Ni}^{2+}, \mathrm{Cu}^{2+}$ & $\mathrm{CH}_{3}$ & $\mathrm{H}$ & $\mathrm{CH}_{3}$ & $\mathrm{dmPz}$ & 32 \\
\hline $\begin{array}{l}\mathrm{Co}^{2+}, \mathrm{Co}^{3+}, \mathrm{Ni}^{2+}, \\
\mathrm{Cu}^{2+}, \mathrm{Zn}^{2+}, \mathrm{Cd}^{2+}\end{array}$ & $\mathrm{H}$ & $\mathrm{H}$ & $\mathrm{H}$ & $\mathrm{Pz}$ & 33 \\
\hline $\begin{array}{l}\mathrm{Co}^{2+}, \mathrm{Co}^{3+}, \mathrm{Ni}^{2+}, \\
\mathrm{Cu}^{2+}, \mathrm{Zn}^{2+}, \mathrm{Cd}^{2+}\end{array}$ & $\mathrm{H}$ & $\mathrm{Cl}$ & $\mathrm{H}$ & $\mathrm{clPz}$ & 34,35 \\
\hline $\begin{array}{l}\mathrm{Co}^{2+}, \mathrm{Co}^{3+}, \mathrm{Ni}^{2+} \\
\mathrm{Cu}^{2+}, \mathrm{Zn}^{2+}, \mathrm{Cd}^{2+}\end{array}$ & $\mathrm{H}$ & $\mathrm{Br}$ & $\mathrm{H}$ & brPz & 34,35 \\
\hline $\begin{array}{l}\mathrm{Co}^{2+}, \mathrm{Co}^{3+}, \mathrm{Ni}^{2+}, \\
\mathrm{Cu}^{2+}, \mathrm{Zn}^{2+}, \mathrm{Cd}^{2+}\end{array}$ & $\mathrm{H}$ & I & $\mathrm{H}$ & $\mathrm{iPz}$ & 34,35 \\
\hline $\begin{array}{l}\mathrm{Co}^{3+}, \mathrm{Cu}^{2+}, \mathrm{Zn}^{2+} \\
\mathrm{Cd}^{2+}, \mathrm{Ag}^{+}\end{array}$ & $\mathrm{H}$ & $\mathrm{NO}_{2}$ & $\mathrm{H}$ & $\mathrm{nPz}$ & 36 \\
\hline $\begin{array}{l}\mathrm{Co}^{2+}, \mathrm{Ni}^{2+}, \mathrm{Cu}^{2+} \\
\mathrm{Zn}^{2+}, \mathrm{Cd}^{2+}, \mathrm{Ag}^{+}\end{array}$ & $\mathrm{H}$ & $\begin{array}{l}\mathrm{CH}_{3} \\
37\end{array}$ & $\mathrm{H}$ & $4-\mathrm{mPz}$ & \\
\hline $\begin{array}{l}\mathrm{Co}^{2+}, \mathrm{Cu}^{2+}, \mathrm{Zn}^{2+} \\
\mathrm{Ag}^{+}\end{array}$ & $\mathrm{CH}_{3}$ & $\mathrm{H}$ & $\mathrm{H}$ & $3-\mathrm{mPz}$ & 37 \\
\hline $\mathrm{Co}^{2+}, \mathrm{Zn}^{2+}, \mathrm{Ag}^{+}$ & $\mathrm{CH}_{3}$ & $\mathrm{H}$ & $\mathrm{CH}_{3}$ & $\mathrm{dmPz}$ & 37 \\
\hline $\mathrm{Cu}^{2+}$ & $\mathrm{H}$ & $\mathrm{H}$ & $\mathrm{H}$ & $\mathrm{Pz}$ & 38,39 \\
\hline $\mathrm{Cu}^{2+}$ & $\mathrm{H}$ & $\mathrm{Cl}$ & $\mathrm{H}$ & $\mathrm{clPz}$ & 39 \\
\hline $\mathrm{Cu}^{2+}$ & $\mathrm{H}$ & $\mathrm{Br}$ & $\mathrm{H}$ & $\mathrm{brPz}$ & 39 \\
\hline $\mathrm{Cu}^{2+}$ & $\mathrm{H}$ & $\mathrm{CH}_{3}$ & $\mathrm{H}$ & $4-\mathrm{mPz}$ & 39 \\
\hline $\mathrm{Pd}^{2+}$ & $\mathrm{H}$ & $\mathrm{H}$ & $\mathrm{H}$ & $\mathrm{Pz}$ & 40 \\
\hline $\mathrm{Pd}^{2+}$ & $\mathrm{H}$ & $\mathrm{CH}_{3}$ & $\mathrm{H}$ & $4-\mathrm{mPz}$ & 40 \\
\hline $\mathrm{Pd}^{2+}$ & $\mathrm{H}$ & I & $\mathrm{H}$ & $\mathrm{iPz}$ & 40 \\
\hline $\mathrm{Pd}^{2+}$ & $\mathrm{CH}_{3}$ & $\mathrm{H}$ & $\mathrm{CH}_{3}$ & $\mathrm{dmPz}$ & 40 \\
\hline $\mathrm{Cu}^{+}, \mathrm{Ag}^{+}$ & $\mathrm{H}$ & $\mathrm{H}$ & $\mathrm{H}$ & $\mathrm{Pz}$ & 41 \\
\hline $\mathrm{Zn}^{2+}, \mathrm{Cd}^{2+}, \mathrm{Hg}^{+2}$ & $\mathrm{H}$ & $\mathrm{H}$ & $\mathrm{H}$ & $\mathrm{Pz}$ & 42 \\
\hline $\begin{array}{l}\mathrm{Co}^{2+}, \mathrm{Co}^{3+}, \mathrm{Ni}^{2+} \\
\mathrm{Fe}^{3+}\end{array}$ & $\mathrm{H}$ & $\mathrm{H}$ & $\mathrm{H}$ & $\mathrm{Pz}$ & 43 \\
\hline
\end{tabular}

$\left[\mathrm{Cu}(\mu-\mathrm{Pz})_{2}\right]_{\mathrm{n}}$, por exemplo, foi obtido na forma de um sólido esverdeado, pela reação de estado sólido entre cobre metálico e pirazol fundido, na presença de fluxo contínuo de ar.

Polímeros pirazólicos de Pd(II) foram, pela primeira vez, sintetizados por nosso grupo de pesquisa ${ }^{40}$ (Tabela 3). Verificou-se, também, neste trabalho a influência da posição do substituinte do anel pirazólico na estabilidade térmica das espécies polinucleares.

A difração de raios-X pelo método do pó tornou-se uma poderosa ferramenta na determinação estrutural de polímeros de coordenação, devido à disponibilidade de equipamentos avançados de alta resolução e de fontes de radiação intensa (síncroton), bem como pelo desenvolvimento de técnicas numéricas e de algoritmos para manipulação dos difratogramas digitalizados. Dentro dessa perspectiva, Masciocchi et al. ${ }^{41-43}$ caracterizaram cristalograficamente uma série de polímeros de coordenação de fórmula geral $[\mathrm{M}(\mu$ $\left.\mathrm{Pz})_{1-3}\right]_{\mathrm{n}}$ (Tabela 3), empregando a técnica de determinação estrutural $a b$ initio a partir da difração de raios-X pelo método do pó, e refinadas pelo método de Rietveld. A Figura 13 representa a fórmula estrutural da espécie polimérica de cobre $[\mathrm{Cu}(\mu-\mathrm{Pz})]_{\mathrm{n}}$.

Observando a Figura 13, nota-se que há uma tendência de polímeros pirazólicos de coordenação homolépticos em formar cadeias unidimensionais lineares. No entanto, a introdução de coligantes na esfera de coordenação do centro metálico pode modificar a topologia do polímero $1 \mathrm{D}$ ou conferir um aumento na dimensionalidade da arquitetura. Por exemplo, foi verificado pelo nosso grupo que a auto-montagem entre o íon $\mathrm{Pd}(\mathrm{II})$, pirazol $(\mathrm{HPz})$ e o pseudo-haleto azida $\left(\mathrm{N}_{3}{ }^{-}\right)$conduz à formação de um polímero de coordenação de topologia senoidal, ${ }^{44}$ conforme ilustrado na Figura 14.

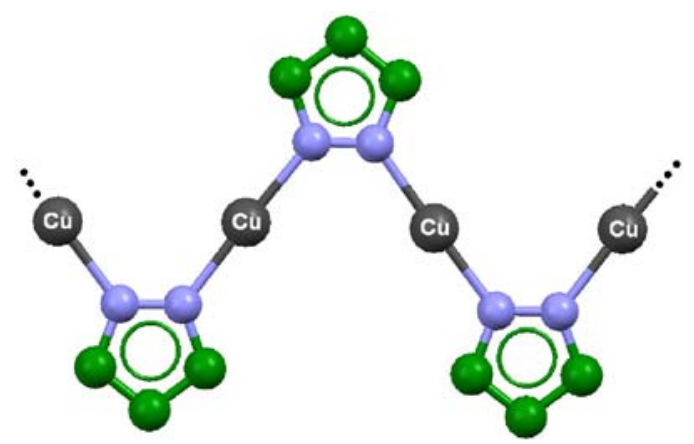

Figura 13. Fórmula estrutural do polímero $[\mathrm{Cu}(\mu-\mathrm{Pz})]_{n}$

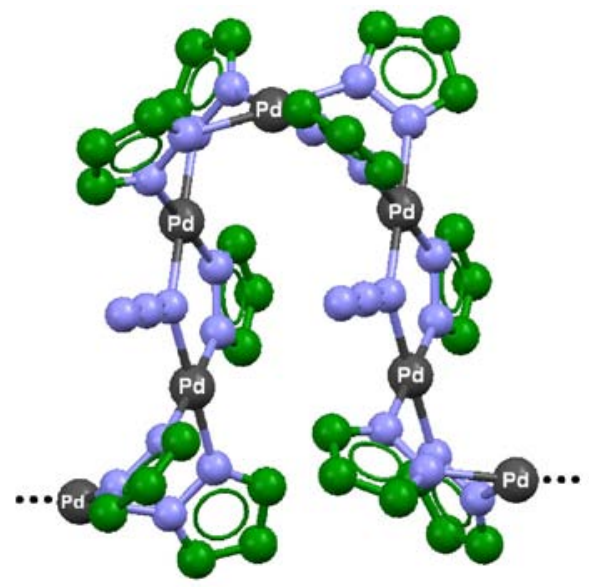

Figura 14. Fórmula estrutural do polímero de coordenação $\left[P d_{3}\left(\mu-N_{3}\right)(\mu\right.$ $\left.P z)_{5}\right]_{n}$

A capacidade de ligantes carboxilatos de atuarem como pontes entre centros metálicos também tem sido explorada na obtenção de polímeros de coordenação contendo pirazóis. A partir da auto-montagem das unidades trinucleares $\left[\mathrm{Cu}_{3}\left(\mu_{3}-\mathrm{OH}\right)(\mu-\mathrm{Pz})_{3}(\mathrm{RCOO})_{2}\right](\mathrm{R}=$ $\left.\mathrm{H}, \mathrm{C}_{2} \mathrm{H}_{5}\right)$, Monari et al. ${ }^{45}$ prepararam polímeros de coordenação unie bidimensionais, respectivamente. Esses autores também obtiveram sucesso na obtenção dos polímeros $\left[\mathrm{M}\left(\mu-\mathrm{CH}_{3} \mathrm{COO}\right)(\mu-\mathrm{Pz})\right]_{\mathrm{n}}(\mathrm{M}$ $=\mathrm{Cd}, \mathrm{Zn})$ cujas estruturas revelam a formação de camadas bidimensionais via grupos acetato e pirazolato exobidentados. . $^{46,47}$ Quando $\mathrm{Hg}$ (II) é empregado como íon metálico, ${ }^{47}$ ocorre a formação dos trímeros $\left[\mathrm{Hg}_{3}(\mu-\mathrm{Pz})_{3}\right]$ que se interconectam através de grupos acetato, dando origem à espécie $\left[\mathrm{Hg}_{3}\left(\mu-\mathrm{CH}_{3} \mathrm{COO}\right)_{3}(\mu-\mathrm{Pz})_{3}\right]_{\mathrm{n}} \mathrm{de}$ dimensionalidade 3D.

\section{Espécies supramoleculares não covalentes}

A auto-montagem de compostos pirazólicos de metais de transição via interações não covalentes tem originado uma série de espécies supramoleculares com interessantes arquiteturas multidimensionais. As forças intermoleculares mais utilizadas para esse propósito consistem primordialmente em ligações de hidrogênio e, em menor escala, interações do tipo C-H $\cdots \pi$ e empilhamento $\pi \cdots \pi$. Sob o ponto de vista da Engenharia de Cristais, para que se possa controlar a auto-montagem molecular dentro do cristal, é de importância vital a identificação, utilização e compreensão detalhada dos síntons supramoleculares que governam a orientação, o reconhecimento e a associação dos blocos de montagens no estado sólido.

Segundo Desiraju, ${ }^{48}$ síntons supramoleculares são definidos como arranjos espaciais de interações intermoleculares que incorporam as características de reconhecimento geométrico e químico das molé- 
culas. Uma abordagem alternativa para esse mesmo conceito envolve a definição de Broder et al.. ${ }^{49}$ Segundo esses autores, síntons supramoleculares são descritos como uma combinação de grupos doadores e aceitadores complementares que se reconhecem entre si ao nível molecular e são capazes de gerar interações supramoleculares reprodutíveis e bem definidas no estado sólido (ver a seguir).

\section{Ligações de hidrogênio}

Ligações de hidrogênio são definidas, segundo Etter, ${ }^{50}$ como interações que direcionam a associação de um átomo de hidrogênio ligado covalentemente a um ou mais átomos, grupos de átomos ou moléculas em uma estrutura agregada estável o suficiente a ponto de ser considerada como uma espécie química independente. Devido à força e direcionalidade das ligações de hidrogênio, elas estão presentes na maioria dos síntons como "cimento supramolecular". ${ }^{50}$

Em particular, os ligantes pirazólicos destacam-se por conter em sua estrutura um átomo de nitrogênio pirrólico capaz de atuar como doador de ligação de hidrogênio. O reconhecimento molecular deste grupo $\mathrm{N}-\mathrm{H}$ frente a aceitadores de ligações de hidrogênio desempenha um papel relevante na formação de compostos supramoleculares. Dentre esses aceitadores de hidrogênio, destacam-se os pseudo-haletos, os haletos e os carboxilatos.

No que diz respeito aos pseudo-haletos - que compreendem os ânions poliatômicos azida, tiocianato, isotiocianato, entre outros uma variedade de síntons supramoleculares podem ser formados a partir de ligações de hidrogênio entre o grupo $\mathrm{N}-\mathrm{H}$ do pirazol e o grupo aceitador presente nesses ligantes..$^{51-57} \mathrm{~A}$ Tabela 4 mostra as supramoléculas auto-montadas a partir da ligação de hidrogênio entre pirazóis coordenados e pseudo-haletos.

Tabela 4. Supramoléculas pirazólicas auto-montadas por complexos de fórmula geral $\left[\mathrm{MX}_{2} \mathrm{~L}_{4-\mathrm{x}}\right](\mathrm{X}=$ pseudo-haletos; $\mathrm{x}=0,2)$

\begin{tabular}{lcccc}
\hline $\begin{array}{l}\text { Blocos de } \\
\text { montagens }\end{array}$ & $\mathrm{L}$ & $\begin{array}{c}\text { Geometria de } \\
\text { coordenação }\end{array}$ & Sínton & Ref. \\
\hline$\left[\mathrm{Mn}(\mathrm{NCS})_{2} \mathrm{~L}_{4}\right]$ & $\mathrm{HPz}$ & octaédrica & $(\mathrm{N}-\mathrm{H})_{2} \cdots \mathrm{SCN}$ & 51 \\
{$\left[\mathrm{Ni}(\mathrm{NCS})_{2} \mathrm{~L}_{4}\right]$} & $\mathrm{HfPz}$ & octaédrica & $(\mathrm{N}-\mathrm{H})_{2} \cdots \mathrm{SCN}$ & 52 \\
{$\left[\mathrm{Ni}(\mathrm{NCS})_{2} \mathrm{~L}_{4}\right]$} & $\mathrm{HbPz}$ & octaédrica & $(\mathrm{N}-\mathrm{H})_{2} \cdots \mathrm{SCN}$ & 52 \\
{$\left[\mathrm{Co}(\mathrm{NCS})_{2} \mathrm{~L}_{4}\right]$} & $\mathrm{HPz}$ & octaédrica & $(\mathrm{N}-\mathrm{H})_{2} \cdots \mathrm{SCN}$ & 53 \\
{$\left[\mathrm{Ni}(\mathrm{NCS})_{2} \mathrm{~L}_{4}\right]$} & $\mathrm{HPz}$ & octaédrica & $(\mathrm{N}-\mathrm{H})_{2} \cdots \mathrm{SCN}$ & 53 \\
{$\left[\mathrm{Ni}\left(\mathrm{N}_{3}\right)_{2} \mathrm{~L}_{4}\right]$} & $\mathrm{HPz}$ & octaédrica & $\mathrm{N}-\mathrm{H} \cdots \mathrm{NNN}$ & 54 \\
{$\left[\mathrm{Ni}\left(\mathrm{N}_{3}\right)_{2} \mathrm{~L}_{4}\right]$} & $\mathrm{HmPz}$ & octaédrica & $\mathrm{N}-\mathrm{H} \cdots \mathrm{NNN}$ & 54 \\
{$\left[\mathrm{Mn}\left(\mathrm{N}_{3}\right)_{2} \mathrm{~L}_{4}\right]$} & $\mathrm{HPz}$ & octaédrica & $\mathrm{N}-\mathrm{H} \cdots \mathrm{NNN}$ & 55 \\
{$\left[\mathrm{Mn}\left(\mathrm{N}_{3}\right)_{2} \mathrm{~L}_{4}\right]$} & $\mathrm{HbPz}$ & octaédrica & $\mathrm{N}-\mathrm{H} \cdots \mathrm{NNN}$ & 56 \\
{$\left[\mathrm{Co}\left(\mathrm{N}_{3}\right)_{2} \mathrm{~L}_{4}\right]$} & $\mathrm{HbPz}$ & octaédrica & $\mathrm{N}-\mathrm{H} \cdots \mathrm{NNN}$ & 56 \\
{$\left[\mathrm{Pd}\left(\mathrm{SCN}_{2}\right)_{2} \mathrm{~L}_{2}\right]$} & $\mathrm{HdmPz}$ & quadrado & $\mathrm{N}-\mathrm{H} \cdots \mathrm{NCS}$ & 57 \\
& & -planar & & \\
{$\left[\mathrm{Pd}\left(\mathrm{N}_{3}\right)_{2} \mathrm{~L}_{2}\right]$} & $\mathrm{HdmPz}$ & quadrado & $\mathrm{N}-\mathrm{H} \cdots \mathrm{NCS}$ & 57 \\
& & -planar & & \\
\hline & & & &
\end{tabular}

A título de ilustração, a Figura 15 representa dois tipos de síntons distintos, obtidos através de interações do tipo N-H...SCN.

De acordo com a literatura, ${ }^{58}$ a freqüência em que ocorre um determinado sínton em estruturas cristalinas conhecidas indica sua "robustez". Assim, a ocorrência do sínton (N-H) $\cdots$. SCN nos empacotamentos cristalinos da maioria das moléculas do tipo $\left[\mathrm{M}(\mathrm{NCS})_{2} \mathrm{~L}_{4}\right][\mathrm{L}=\text { ligantes pirazólicos }]^{51-53}$ confirma a robustez do padrão de reconhecimento molecular do tipo $R_{2}{ }_{2}(8) .{ }^{59,60}$

A Figura 16 ilustra a cadeia unidimensional formada por monômeros do tipo $\left[\mathrm{M}(\mathrm{NCS})_{2} \mathrm{~L}_{4}\right]$ que se organizam segundo o mesmo padrão de reconhecimento molecular de dois grupos $\mathrm{N}-\mathrm{H}$ de ligantes pirazólicos, de uma molécula, pelo enxofre terminal de um isotiocianato da molécula adjacente.

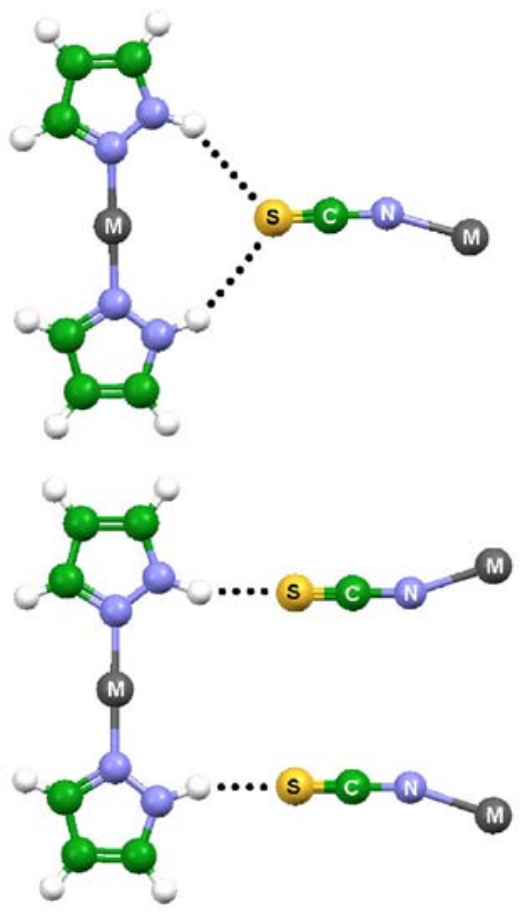

Figura 15. Síntons supramoleculares resultantes de ligações de hidrogênio entre $\mathrm{N}-\mathrm{H}$ pirrólico e isotiocianato

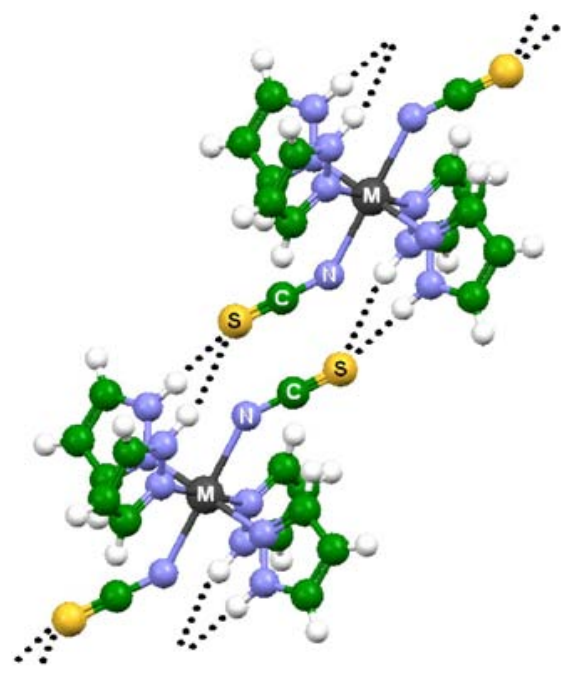

Figura 16. Representação da auto-montagem para os complexos octaédricos $\left[\mathrm{M}(\mathrm{NCS})_{2}(\mathrm{HPz})_{4}\right](\mathrm{M}=\mathrm{Co}, \mathrm{Ni})$

A natureza do centro metálico e a geometria de coordenação do complexo são fatores que influem substancialmente no tipo de sínton formado. Por exemplo, o composto quadrado-planar $\left[\mathrm{Pd}(\mathrm{SCN})_{2}(\mathrm{HdmPz})_{2}\right]$ organiza-se a partir do reconhecimento molecular do tipo $R_{2}^{2}(14)$, via ligações de hidrogênio N-H...NCS, resultando na fita supramolecular ${ }^{57}$ mostrada na Figura 17.

Espécies supramoleculares pirazólicas contendo cloro-complexos como blocos de montagens também são encontradas na literatura, embora em menor frequiência quando comparadas a sistemas análogos apresentando pseudo-haletos como aceitadores de ligação de hidrogênio. ${ }^{61-63}$

A partir da interação intermolecular $\mathrm{N}-\mathrm{H} \cdots \mathrm{Cl}$, diferentes síntons supramoleculares podem ser gerados em função da configuração geométrica do bloco de montagem usado. ${ }^{61,62}$ De fato, o 


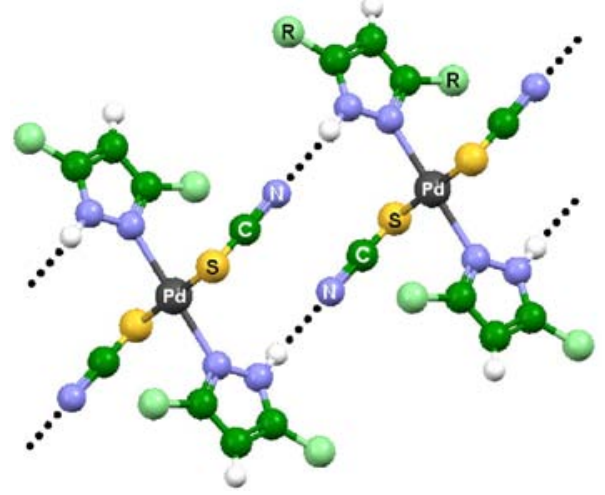

Figura 17. Representação da auto-montagem para o complexo quadradoplanar $\left[\mathrm{Pd}(\mathrm{SCN})_{2}(\mathrm{HdmPz})_{2}\right](\mathrm{R}=\mathrm{Me})$

empacotamento cristalino do complexo cis- $\left[\mathrm{PtCl}_{2}(\mathrm{HPz})_{2}\right]$ consiste em uma fita supramolecular na qual as unidades monoméricas estão associadas via ligações de hidrogênio do padrão $R_{2}{ }_{2}(10)$, como ilustrado na Figura 18. Por outro lado, o composto análogo de configuração trans sofre auto-montagem, originando uma cadeia linear formada pelas unidades trans- $\left[\mathrm{PtCl}_{2}(\mathrm{HPz})_{2}\right]$ empilhadas, umas sobre as outras, e orientadas helicoidalmente.

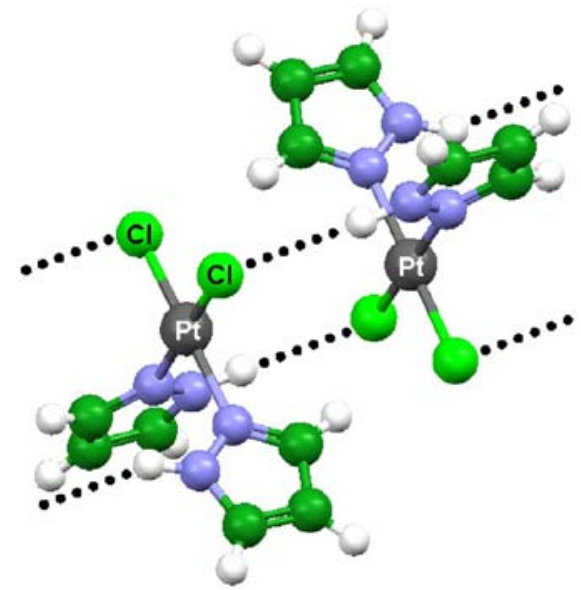

Figura 18. Representação da auto-montagem do complexo cis- $\left[\mathrm{PtCl}_{2}(\mathrm{HPz})_{2}\right]$

Trabalhos envolvendo supramoléculas construídas a partir de ligações de hidrogênio entre o grupo N-H de ligantes pirazólicos e os demais haletos da série $\left(\mathrm{F}^{-}, \mathrm{Br}, \mathrm{I}^{-}\right)$como aceitadores de ligação de hidrogênio ainda não foram descritos na literatura.

Nos últimos anos, atenção também tem sido dada à construção de metalossupramoléculas pirazólicas ${ }^{46,64-66}$ utilizando grupos carboxilatos como aceitadores de ligação de hidrogênio.

Fitas supramoleculares unidimensionais ${ }^{46}$ são formadas a partir da associação espontânea entre os blocos de montagens diméricos $\left[\mathrm{Zn}\left(\mathrm{CH}_{3} \mathrm{COO}\right)(\mu-\mathrm{pz})(\mathrm{HPz})\right]_{2}$ através de ligações de hidrogênio envolvendo o átomo de oxigênio não coordenado do ânion acetato e o grupo N-H do pirazol de um dímero vizinho, conforme representado na Figura 19.

Com relação a grupos dicarboxilatos como co-ligantes, observase uma tendência na formação de polímeros de coordenação 1D nos quais esses ânions atuam como pontes entre os centros metálicos. No entanto, a presença de ligantes pirazólicos monodentados nesses compostos possibilita a formação de espécies supramoleculares de dimensionalidade superior. ${ }^{64,65}$ Sendo assim, nos compostos $\left[\mathrm{Cu}(\mathrm{tcft})(\mathrm{HPz})_{2}\right]$ e $\left[\mathrm{Ni}(\mathrm{tf})(\mathrm{HPz})_{4}\right]$, os ligantes pirazólicos atuam como

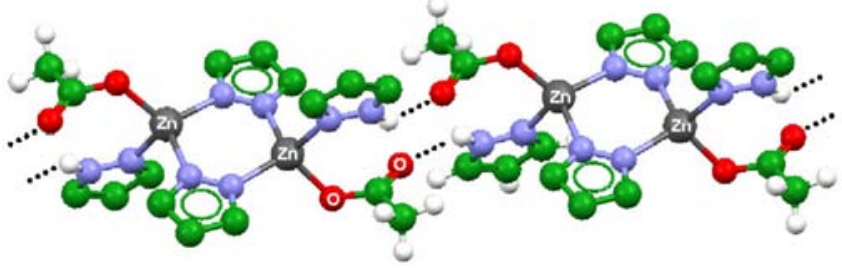

Figura 19. Representação das ligações de hidrogênio no dímero $\left[\mathrm{Zn}\left(\mathrm{CH}_{3} \mathrm{COO}\right)(\mu-\mathrm{pz})(\mathrm{HPz})\right]_{2}$

doadores de ligação de hidrogênio frente aos grupos carboxilatos de uma cadeia adjacente, permitindo a auto-organização de arquiteturas bi- e tridimensionais, respectivamente.

Forniés et $a l .{ }^{66}$ descreveram recentemente a preparação do cristal $\left[\mathrm{Pd}(\mathrm{HdmPz})_{4}\right](\mathrm{N} \text {-acetilglicinato })_{2}$, no qual os ânions $\mathrm{N}$-acetilglicinato $\left(\mathrm{O}_{2} \mathrm{CCH}_{2} \mathrm{NHCOCH}_{3}\right)$ sofrem auto-montagem através de ligações de hidrogênio do tipo $\mathrm{N}-\mathrm{H} \cdots \mathrm{O}$ formando cadeias lineares infinitas. O reconhecimento molecular entre os grupos carboxilatos do ânion e os grupos $\mathrm{N}-\mathrm{H}$ dos ligantes pirazólicos do cátion $\left[\mathrm{Pd}(\mathrm{HdmPz})_{4}\right]^{2+}$ gera $\mathrm{o}$ sínton supramolecular $(\mathrm{N}-\mathrm{H})_{2} \cdots \mathrm{OOC}$ que, por sua vez, é responsável pela auto-organização em uma rede 2D.

\section{Outras interações não-covalentes}

Recentemente, a literatura tem mostrado que forças nãocovalentes mais fracas que as ligações de hidrogênio convencionais também desempenham um papel importante em processos de auto-organização ou reconhecimento molecular na Química e na Bioquímica. ${ }^{31,67}$ As interações C-H $\cdots \pi$ e $\pi \cdots \pi$ são algumas dessas forças responsáveis pela estabilização do empacotamento de cristais moleculares orgânicos, uma vez que grupos C-H e anéis aromáticos são abundantes na estrutura dessas substâncias. Não obstante a baixa intensidade dessas forças $\left(\sim 1-2 \mathrm{~kJ} \mathrm{~mol}^{-1}\right)$, o efeito cooperativo dessas interações intermoleculares não é desprezível.

No que concerne a compostos de coordenação, ainda há poucos trabalhos focados na questão de como complexos ou polímeros de coordenação são empacotados na rede cristalina. Embora haja evidências experimentais de interações $\mathrm{C}-\mathrm{H} \cdots \pi$ e $\pi \cdots \pi$ em estruturas de alguns complexos, sua descrição estrutural não é bem documentada, ainda que seja de fundamental importância para o desenvolvimento da Química Inorgânica Supramolecular.

Particularmente, por se tratarem de ligantes heterocíclicos aromáticos, os pirazóis desempenham um papel importante na agregação molecular não somente pela possibilidade de formação de ligações de hidrogênio, como também dessas outras interações não-covalentes.

Considerando o critério estabelecido por Janiak, ${ }^{31}$ os anéis pirazólicos presentes na estrutura molecular de complexos podem adotar diferentes orientações, de modo a maximizar a estabilização de seus empacotamentos no cristal. Como mostrado na Figura 20, há duas situações-limite no que se refere ao arranjo dos anéis

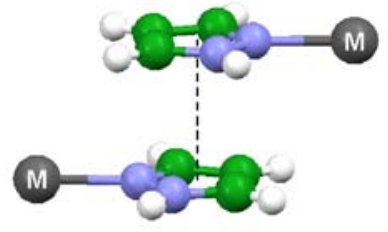

face - to - face

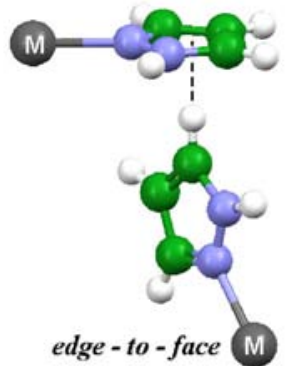

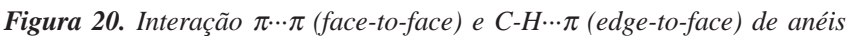
pirazólicos coordenados $(M=$ metal $)$ 
aromáticos: um paralelo (face-to-face ou empilhamento $\pi \cdots \pi$ ) e o outro perpendicular (edge-to-face ou conformação-T). Embora o termo "empilhamento $\pi \cdots \pi$ " seja algumas vezes indiscriminadamente utilizado, é mais apropriado descrever como interação $\mathrm{C}-\mathrm{H} \cdots \pi$ (edge-to-face) quando os planos dos anéis aromáticos se encontrarem orientados em um ângulo próximo de $90^{\circ}$ no retículo cristalino do complexo. Por outro lado, para que haja um empilhamento $\pi \cdots \pi$, não é necessária a ocorrência de um alinhamento facial perfeito dos anéis aromáticos, podendo haver certo deslizamento de um anel com relação ao outro, que é, na verdade, observado na grande maioria dos casos. Vale dizer, também, que nesse empilhamento de grupos aromáticos, a distância interplanar varia de 3,3 a $3,8 \AA$. Na realidade, as forças intermoleculares que atuam entre os anéis pirazólicos - e que são responsáveis pela estabilização do empilhamento $\pi \cdots \pi$ - consistem em atrações do tipo van der Waals de caráter dipolo-dipolo.

Compostos octaédricos do tipo $\left[\mathrm{MX}_{2}(\mathrm{HPz})_{4}\right](\mathrm{M}=\mathrm{Co}, \mathrm{Ni} ; \mathrm{X}=$ $\left.\mathrm{NCS}, \mathrm{N}_{3}\right)^{53,54}$ sofrem auto-montagem mediante interações $\mathrm{C}-\mathrm{H} \cdots \pi$ e $\pi \cdots \pi$ envolvendo anéis pirazólicos de unidades adjacentes, conforme ilustrado na Figura 21.

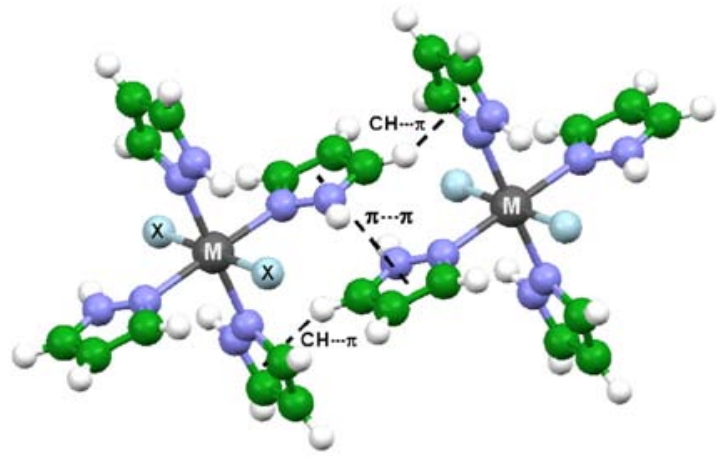

Figura 21. Interações $C-H \cdots \pi$ e $\pi \cdots \pi$ em sistemas $\left[\mathrm{MX}_{2}(\mathrm{HPz})_{4}\right](\mathrm{M}=\mathrm{Co}, \mathrm{Ni}$; $X=N C S, N_{3}$ )

Nosso grupo de pesquisa também constatou recentemente que forças intermoleculares do tipo $\pi \cdots \pi$ são responsáveis pela autoorganização de cadeias $1 \mathrm{D}$ do polímero de coordenação $[\mathrm{Cd}(\mu-$ $\left.1,3-\mathrm{NCS})_{2}(\mathrm{HPz})_{2}\right]_{\mathrm{n}}$ em folhas supramoleculares bidimensionais. ${ }^{68}$ Perfil semelhante de interação foi observado no trabalho de Hong et al. ${ }^{65}$ no qual as cadeias do polímero $\left[\mathrm{Ni}(\mathrm{tf})(\mathrm{HPz})_{4}\right]_{\mathrm{n}}$ se estruturam em uma arquitetura tridimensional via contato $\pi \cdots \pi$.

Contatos $\pi \cdots \pi$ também podem se estabelecer entre pirazóis e outros anéis aromáticos. No trabalho de Yilmaz et al. ${ }^{69}$ o polímero de coordenação $\left[\mathrm{Ag}(\mathrm{sac})(\mathrm{HPz})\left(\mu-\mathrm{H}_{2} \mathrm{O}\right)\right]_{n}$ se auto-organiza em um arranjo supramolecular 3D através da interação $\pi_{\mathrm{HPz}} \cdots \pi_{\mathrm{sac}}$ e ligações de hidrogênio do tipo N-H…O.

Cabe ressaltar que a força de atração $\mathrm{C}-\mathrm{H} \cdots \pi$ não se limita apenas à interação entre grupos aromáticos. Ainda que escassos, há relatos na literatura sobre, por exemplo, cristais moleculares ${ }^{70}$ cujos blocos de montagens $\left[\mathrm{Mn}\left(\mathrm{X}-\mathrm{C}_{6} \mathrm{H}_{4} \mathrm{COO}\right)_{2}(\mathrm{HdpPz})_{4}\right]\left(\mathrm{X}=\mathrm{NO}_{2}, \mathrm{~F}\right)$ são auto-montados através da interação entre a nuvem eletrônica $\pi$ do ligante carboxilato e um grupo metílico da ramificação iso-propil do ligante pirazólico de uma molécula adjacente.

\section{QUÍMICA HÓSPEDE-HOSPEDEIRO DE COMPLEXOS PIRAZÓLICOS}

A Química Hóspede-Hospedeiro é a área da Química que envolve a investigação de associações moleculares orgânicas e/ou inorgânicas altamente estruturadas constituídas por, no mínimo, um componente hospedeiro e um componente hóspede, unidos por forças não-covalentes. ${ }^{71}$ A Química Hóspede-Hospedeiro tem como essência o princípio do reconhecimento molecular entre os componentes que constituem estas espécies discretas oligomoleculares, também conhecidas como supermoléculas. ${ }^{20}$

No que diz respeito a sistemas hóspede-hospedeiro envolvendo complexos pirazólicos, há uma tendência na formação de supermoléculas nas quais complexos catiônicos atuam como hospedeiros de grupos aniônicos. Os trabalhos de Halcrow et al., ${ }^{72}$ por exemplo, evidenciam a capacidade do cátion-complexo $\left[\mathrm{ZnCl}(\mathrm{HbPz})_{3}\right]^{+}$em atuar como pinças moleculares frente a várias espécies aniônicas, conforme ilustrado na Figura 22. A configuração dos anéis pirazólicos na estrutura molecular do hospedeiro origina uma cavidade onde os grupos pirrólicos apontam para uma mesma face, permitindo, então, o reconhecimento molecular via ligação de hidrogênio $\mathrm{N}-\mathrm{H} \cdots \mathrm{X}\left(\mathrm{X}=\mathrm{Cl}, \mathrm{Br}, \mathrm{I}, \mathrm{BF}_{4}, \mathrm{ClO}_{4}, \mathrm{NO}_{3}, \mathrm{CF}_{3} \mathrm{SO}_{3}\right.$, $\mathrm{PF}_{6}$ ). A substituição do cloro por mais um anel pirazólico na estrutura desse hospedeiro ${ }^{73}$ gera o cátion $\left[\mathrm{Zn}(\mathrm{HbPz})_{4}\right]^{+2}$, capaz de interagir com grupos $\mathrm{NO}_{3}^{-}$via ligações de hidrogênio do tipo $R_{2}^{1}(8)$.

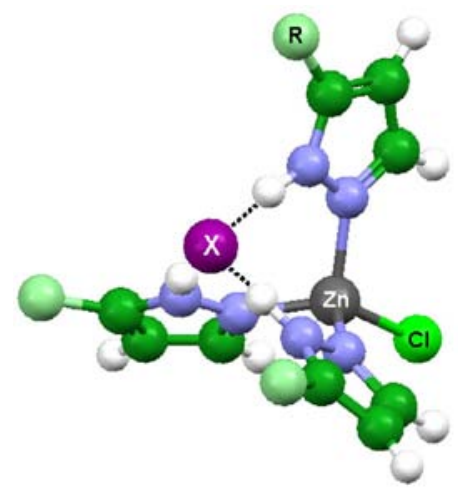

Figura 22. Formação de supermoléculas envolvendo o hospedeiro $\left[\mathrm{ZnCl}(\mathrm{HbPz})_{3}\right]^{+}$e ânions $\mathrm{X}^{-}\left(\mathrm{R}={ }^{\mathrm{t}} \mathrm{Bu} ; \mathrm{X}=\mathrm{Cl}, \mathrm{Br}, \mathrm{I}, \mathrm{BF}_{4}, \mathrm{ClO}_{4} \mathrm{NO}_{3}\right.$, $\mathrm{CF}_{3} \mathrm{SO}_{3}, \mathrm{PF}_{6}$ )

A geometria de coordenação do íon metálico exerce uma influência significativa no padrão de ligação de hidrogênio. Assim, o tipo de ligação de hidrogênio $R_{2}^{1}(8)$ observado nas supermoléculas ${ }^{74}$ $\left[\operatorname{Re}(\mathrm{CO})_{3}(\mathrm{~L})_{3}\right]^{+} \supset \mathrm{X}^{-}\left(\mathrm{L}=\mathrm{HdmPz}, \mathrm{HbPz} ; \mathrm{X}=\mathrm{Cl}, \mathrm{Br}, \mathrm{I}, \mathrm{HSO}_{4}, \mathrm{ReO}_{4}\right.$, $\mathrm{NO}_{3}$ ) difere substancialmente do padrão observado para sistemas de coordenação linear. Cano et al. ${ }^{75}$ obtiveram compostos de prata e ouro univalentes, de fórmula geral $\left[\mathrm{M}(\mathrm{L})_{2}\right]^{+}(\mathrm{L}=\mathrm{HdboxfPz}$, $\mathrm{HdmnPz}$ ), que se associam com os ânions $\mathrm{CH}_{3} \mathrm{C}_{6} \mathrm{H}_{4} \mathrm{SO}_{3}^{-}, \mathrm{BF}_{4}^{-} \mathrm{e}$ $\mathrm{CF}_{3} \mathrm{SO}_{3}{ }^{-}$pelo padrão $R_{2}^{2}(10)$.

Ligações de hidrogênio envolvendo grupos aniônicos também podem ocorrer entre dois hospedeiros catiônicos. Compostos de paládio(II) de fórmula geral $\left[\mathrm{Pd}\left(\eta^{3} \text {-alil }\right)(\mathrm{L})_{2}\right]^{+}(\mathrm{L}=\mathrm{HdoxfPz}$, $\mathrm{HdmnPz})^{76}$ interagem com os ânions $\mathrm{NO}_{3}^{-} \mathrm{e} \mathrm{BF}_{4}^{-}$(Figura 23) através do padrão de reconhecimento molecular $R_{4}^{4}(20)$.

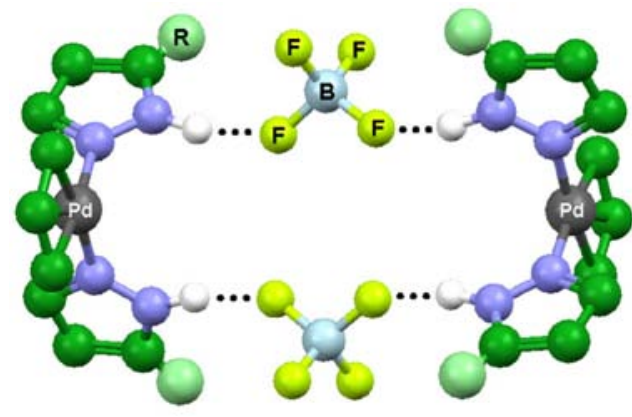

Figura 23. Associação entre $\left[P d\left(\eta^{3}-\text { alil }\right)(H d o x f P z)_{2}\right]^{+}$e $B F_{4}^{-}\{R=3-$ (dodecilóxifenil)\} 


\section{CONCLUSÃO}

Esta revisão teve como objetivo principal apresentar o estadoda-arte da química supramolecular de complexos pirazólicos. Optou-se por enfatizar os aspectos estruturais desta classe de compostos, desde espécies discretas até sistemas de alta dimensionalidade, incluindo também associações moleculares via forças não-covalentes.

É importante salientar que houve, também, a preocupação dos autores em, a partir dos pirazóis como fio condutor do texto, dar uma contribuição didática a temas atuais, ainda não familiares à comunidade científica - e de difícil acesso na língua portuguesa tais como Engenharia de Cristais, síntons supra-moleculares, automontagem, interações não-covalentes, reconhecimento molecular, entre outros.

Neste trabalho ficou constatada, não somente a versatilidade dos pirazóis no que se refere à construção de arquiteturas inorgânicas sustentadas por ligações covalentes, como também sua capacidade em propiciar vários tipos de interações intermoleculares. Interações como ligações de hidrogênio, contatos $\mathrm{C}-\mathrm{H} \cdots \pi$ ou empilhamento $\pi \cdots \pi$ podem, então, ser usadas como cimento supramolecular na união controlada dos blocos de montagens dentro do cristal.

Concluindo, o avanço na investigação do uso de ligantes nitrogenados heterocíclicos na auto-montagem de metalossupramoléculas tem contribuído substancialmente para a Química Supramolecular Inorgânica e Engenharia de Cristais, além de acrescentar novos horizontes no desenvolvimento de novos materiais portadores de propriedades ópticas, magnéticas, mesogênicas e capazes de desempenhar funções tais como armazenamento e separação de gases, troca iônica, materiais microporosos, sensores moleculares, entre outras.

\section{AGRADECIMENTOS}

Ao CNPq, CAPES e FAPESP pelas bolsas e auxílios concedidos.

\section{ABREVIAÇÕES}

$\begin{array}{ll}\mathrm{HPz} & \text { pirazol } \\ \mathrm{HbPz} & \text { 3-terc-butilpirazol } \\ \mathrm{HdoxfPz} & \text { 3-(dodecilóxifenil)pirazol } \\ \mathrm{HfPz} & \text { 3-fenilpirazol } \\ \mathrm{HmPz} & \text { 3-metilpirazol } \\ \mathrm{HdpPz} & \text { 3,5-di-iso-propilpirazol } \\ \mathrm{HdfPz} & \text { 3,5-difenilpirazol } \\ \mathrm{HdmPz} & \text { 3,5-dimetilpirazol } \\ \mathrm{HdboxfPz} & \text { 3,5-bis(4-n-butóxifenil)pirazol } \\ \mathrm{HdmnPz} & \text { 3,5-dimetil-4-nitropirazol } \\ \mathrm{Pz} & \text { ânion pirazolato } \\ \text { aoxfPz } & \text { ânion 3-[(4-alquilóxi)fenil]pirazolato } \\ \mathrm{fPz} & \text { ânion 3-fenilpirazolato } \\ \text { foxfPz } & \text { ânion 3-[(4-fenóxi)fenil]pirazolato } \\ \mathrm{fmPz} & \text { ânion 3-trifluorometilpirazolato } \\ 3-\mathrm{mPz} & \text { ânion 3-metilpirazolato } \\ \text { brPz } & \text { ânion 4-bromopirazolato } \\ \text { clPz } & \text { ânion 4-cloropirazolato } \\ \mathrm{iPz} & \text { ânion 4-iodopirazolato } \\ 4-\mathrm{mPz} & \text { ânion 4-metilpirazolato } \\ \text { nPz } & \text { ânion 4-nitropirazolato } \\ \text { daoxfPz } & \text { ânion 3,5-bis[(4-alquilóxi)fenil]pirazolato } \\ \mathrm{dbPz} & \text { ânion 3,5-di-terc-butilpirazolato } \\ \text { dcbPz } & \text { ânion 3,5-di-carbo-sec-butoxipirazolato } \\ \mathrm{dfPz} & \text { ânion 3,5-difenilpirazolato } \\ \mathrm{ddoxfPz} & \text { ânion 3,5-bis[(3,4-di-decilóxi)fenil]pirazolato } \\ \mathrm{dfoxfPz} & \text { ânion 3,5-bis[(4-fenóxi)fenil]pirazolato } \\ \mathrm{dfmPz} & \text { ânion 3,5-bis(trifluorometil)pirazolato } \\ \mathrm{dmPz} & \text { ânion 3,5-dimetilpirazolato } \\ \text { doPz } & \text { ânion 3,5-dioctilpirazolato } \\ \mathrm{dpPz} & \text { ânion 3,5-di-iso-propilpirazolato } \\ & \end{array}$

$\begin{array}{ll}\text { bfmPz } & \text { ânion 3-terc-butil-5-trifluorometilpirazolato } \\ \text { bpPz } & \text { ânion 3-terc-butil-5-iso-propilpirazolato } \\ \text { dtdoxfPz } & \text { ânion 3-(3,4-di-decilóxi)fenil-5-(3,4,5-tri-decilóxi)fenilpirazolato } \\ \text { fmfPz } & \text { ânion 3-trifluorometil-5-fenilpirazolato } \\ \mathrm{fmmPz} & \text { ânion 3-trifluorometil-5-metilpirazolato } \\ \mathrm{mfPz} & \text { ânion 3-metil-5-fenilpirazolato } \\ \mathrm{dmaPz} & \text { ânion 3,5-dimetil-4-alquilpirazolato } \\ \mathrm{dmodoxbPz} & \text { ânion 3,5-dimetil-4-(3,5-di-octadecilóxi)benzilpirazolato } \\ \mathrm{dmnPz} & \text { ânion 3,5-dimetil-4-nitropirazolato } \\ \mathrm{dpbPz} & \text { ânion 3,5-di-iso-propil-4-bromopirazolato } \\ \mathrm{dpnPz} & \text { ânion 3,5-di-iso-propil-4-nitropirazolato } \\ \mathrm{PPh} & \text { trifenilfosfina } \\ \mathrm{dppf} & \text { difenilfosfinaferroceno } \\ \mathrm{Tf} & \text { ânion tereftalato } \\ \mathrm{Tcft} & \text { ânion tetracloroftalato } \\ \mathrm{Cp} & \text { ânion ciclopentadienilo } \\ \mathrm{Sac} & \text { ânion sacarinato } \\ \mathrm{R} & \text { grupo alquil genérico } \\ \mathrm{Ph} & \text { fenil }\end{array}$

\section{REFERÊNCIAS E NOTAS}

1. Davies, D. T.; Aromatic Heterocyclic Chemistry, Oxford Science Publication: Oxford, 1992, cap. 4.

2. Joule, J. A.; Mills, K.; Smith, G. F.; Heterocyclic Chemistry, $3^{\text {rd }}$ ed., Chapman \& Hall: London, 1995, cap. 22.

3. Elguero, J. Em Comprehensive Heterocyclic Chemistry: The Structure, Reactions, Synthesis and Uses of Heterocyclic Compounds; Katritzky, A. R.; Rees, C. W., eds.; Pergamon Press: Oxford, 1984, v. 5, cap. 4.04.

4. Trofimenko, S.; Chem. Rev. 1972, 72, 497.

5. Trofimenko, S.; Prog. Inorg. Chem. 1986, 34, 115.

6. Sadimenko, A. P.; Basson, S. S; Coord. Chem. Rev. 1996, 147, 247.

7. Ardizzoia, G. A.; La Monica, G.; Prog. Inorg. Chem. 1997, 46, 151.

8. Netto, A. V. G.; Frem, R. C. G.; Mauro, A. E.; Santos, R. H. A; Zoia, J. R.; Trans. Met. Chem. 2002, 27, 279.

9. Fackler Jr., J. P.; Raptis, R. G.; Murray, H. H.; Inorg. Chim. Acta 1992, $193,173$.

10. Yélamos, C.; Heeg, M. J.; Winter, C. H.; Inorg. Chem. 1999, 38, 1871.

11. Pettinari, C.; Marchetti, F.; Cingolani, A.; Troyanov, S. I.; Drozdov, A.; J. Chem. Soc. Dalton Trans. 1998, 3335.

12. Perera, J. R.; Heeg, M. J.; Schlegel, H. B.; Winter, C. H.; J. Am. Chem. Soc. 1999, 121, 4536.

13. Claver, C.; Kalck, P.; Ridmy, M.; Thorez, A.; Oro, L. A.; Pinillos, M. T.; Apreda, M. C.; Cano, F. M.; Foces-Foces, C.; J. Chem. Soc. Dalton Trans. 1988, 1523

14. Li, K.; Darkwa, J.; Guzei, I. A.; Mapolie, S. F.; J. Organomet. Chem. 2002 , 660,108

15. Torralba, M. C.; Cano, M.; Campo, J. A.; Heras, J. V.; Pinilla, E.; Torres, M. R.; Inorg. Chem. Commun. 2002, 5, 887.

16. Kim, S. J.; Kang, S. H.; Park, K.-M.; Kim, H.; Zin, W.-C.; Choi, M.-G.; Kim, K.; Chem. Mater. 1998, 10, 1889; Barberá, J.; Elduque, A.; Giménez, R.; Oro, L. A.; Serrano, J. L.; Angew. Chem., Int. Ed. 1996, 35, 2832.

17. Nomiya, K.; Noguchi, R.; Ohsawa, K.; Tsuda, K.; Oda, M.; J. Inorg. Biochem. 2000, 78, 363.

18. Komeda, S.; Lutz, M.; Spek, A. L.; Chikuma, M.; Reedijk, J.; Inorg. Chem. 2000, 39, 4230.

19. Al-Allaf, T. A. K.; Rashan, L. J.; Asian J. Chem. 1999, 11, 1543.

20. Lehn, J.-M.; Supramolecular Chemistry: Concepts and Perspectives, VCH: Weinheim, 1995.

21. Klein, O.; Aguilar-Parrilla, F.; Lopez, J.M.; Jagerovic, N.; Elguero, J.; Limbach, H.-H.; J. Am. Chem. Soc. 2004, 126, 11718.

22. Ardizzoia, G. A.; Cenini, S.; La Monica, G.; Masciocchi, N.; Maspero, A.; Moret, M.; Inorg. Chem. 1998, 37, 4284; Ehlert, M. K.; Rettig, S. J.; Storr, A.; Thompson, R. C.; Trotter, J.; Can. J. Chem. 1990, 68, 1444; He, J.; Yin, Y.-G.; Wu, T.; Li, D.; Huang, X.-C.; Chem. Commun. 2006, 2845; Dias, H. V. R.; Diyabalanage, H. V. K.; Eldabaja, M. G.; Elbjeirami, O.; Rawashdeh-Omary, M. A.; Omary, M. A.; J. Am. Chem. Soc. 2005, 127, 7489; Raptis, R. G.; Fackler Jr., J. P.; Inorg. Chem. 1988, 27, 4179 ; Fujisawa, K.; Ishikawa, Y.; Miyashita, Y.; Okamoto, K.-I.; Chem. Lett. 2004, 33, 66; Vorontsov, I. I.; Kovalevsky, A. Y.; Chen, Y.-S.; Graber, T.; Gembicky, M.; Novozhilova, I. V.; Omary, M. A.; Coppens, P.; Phys. Rev. Lett. 2005, 94, 193003; Omary, M. A.; Rawashdeh-Omary, M. A.; Gonser, M. W. A.; Elbjeirami, O.; Grimes, T.; Cundari, T. R.; Diyabalanage, H. V. K.; Gamage, C. S. P.; Dias, H. V. R.; Inorg. Chem. 2005, 44, 8200; Dias, H. V. R.; Diyabalanage, H. V. K.; Rawashdeh-Omary, M. A.; Franzman, M. A.; Omary, M. A.; J. Am. Chem. Soc. 2003, 125, 12072. 
23. Masciocchi, N.; Moret, M.; Cairati, P.; Sironi, A.; Ardizzoia, G. A.; La Monica, G.; J. Am. Chem. Soc. 1994, 116, 7668; Murray, H. H.; Raptis, R. G.; Fackler Jr., J. P.; Inorg. Chem. 1988, 27, 26; Mohamed, A. A.; Perez, L. M.; Fackler Jr., J. P.; Inorg. Chim. Acta 2005, 358, 1657; Dias, H. V. R.; Diyabalanage, H. V. K.; Polyhedron 2006, 25, 1655; Chi, Y.; Lay, E.; Chou, T.-Y.; Song, Y.-H.; Carty, A. J.; Chem. Vap. Dep. 2005, 11, 206; Torralba, M. C.; Ovejero, P.; Mayoral, M. J.; Cano, M.; Campo, J. A.; Heras, J. V.; Pinilla, E.; Torres, M. R.; Helv. Chim. Acta 2004, 87, 250.

24. Bovio, B.; Bonati, F.; Banditelli, G.; Inorg. Chim. Acta 1984, 87, 25; Kim, S. J.; Kang, S. H.; Park, K.-M.; Kim, H.; Zin, W.-C.; Choi, M.-G.; Kim, K.; Chem. Mater. 1998, 10, 1889; Barberá, J.; Elduque, A.; Giménez, R.; Oro, L. A.; Serrano, J. L.; Angew. Chem., Int. Ed. 1996, 35, 2832 Kishimura, A.; Yamashita, T.; Aida, T.; J. Am. Chem. Soc. 2005, 127, 179; Yang, G.; Raptis, R. G.; Inorg. Chem. 2003, 42, 261; Sansores, L. E.; Salcedo, R.; Mart1nez, A.; Mireles, N.; J. Mol. Struct.: Theochem. 2006, $763,7$.

25. Baran, P.; Marrero, C. M.; Pérez, S.; Raptis, R. G.; Chem. Commun. 2002, 1012; Umakoshi, K.; Yamauchi, Y.; Nakamiya, K.; Kojima, T.; Yamasaki, M.; Kawano, H.; Onishi, M.; Inorg. Chem. 2003, 42, 3907.

26. Burger, W.; Strähle, J.; Z. Anorg. Allg. Chem. 1985, 529, 111.

27. Ardizzoia, G. A.; Cenini, S.; La Monica, G.; Masciocchi, N.; Moret, M.; Inorg. Chem. 1994, 33, 1458; Maspero, A.; Brenna, S.; Galli, S.; Penoni, A.; J. Organomet. Chem. 2003, 672, 123; Yang, G.; Raptis, R. G.; Inorg. Chim. Acta 2007, 360, 2503; Yang, G.; Raptis, R. G.; Inorg. Chim. Acta 2003, 352, 98.

28. Raptis, R. G.; Murray, H. H.; Fackler Jr., J. P.; J. Chem. Soc., Chem. Commun. 1987, 737.

29. Mezei, G.; Baran, P.; Raptis, R. G.; Angew. Chem., Int. Ed. 2004, 43, 573.

30. Ardizzoia, G. A.; Angaroni, M. A.; La Monica, G.; Cariati, F.; Moret, M.; Masciocchi, N.; Inorg. Chem. 1991, 30, 4347.

31. Janiak, C.; J. Chem. Soc., Dalton Trans. 2000, 3885.

32. Singh, C. B.; Satpathy, S.; Sahoo, B.; J. Inorg. Nucl. Chem. 1973, 35, 3950

33. Vos, J. G.; Groeneveld, W. L.; Inorg. Chim. Acta 1977, 24, 123.

34. Vos, J. G.; Groeneveld, W. L.; Inorg. Chim. Acta 1978, 26, 71.

35. Vos, J. G.; Groeneveld, W. L.; Inorg. Chim. Acta 1978, 27, 173.

36. Nieuwpoort, G.; Vos, J. G.; Groeneveld, W. L.; Inorg. Chim. Acta 1978 $29,117$.

37. Vos, J. G.; Groeneveld, W. L.; Trans. Met. Chem. 1979, 4, 137.

38. Ehlert, M. K.; Rettig, S. J.; Storr, A.; Thompson, R. C.; Trotter, J.; Can. J. Chem. 1989, 67, 1970.

39. Ehlert, M. K.; Rettig, S. J.; Storr, A.; Thompson, R. C.; Trotter, J.; Can. J. Chem. 1991, 69, 432.

40. Netto, A. V. G.; Frem, R. C. G.; Mauro, A. E.; Crespi, M. S.; Zorel Jr., H. E.; J. Therm. Anal. Cal. 2007, 87, 789.

41. Masciocchi, N.; Moret, M.; Cairati, P.; Sironi, A.; Ardizzoia, G. A.; La Monica, G.; J. Am. Chem.Soc. 1994, 116, 7668

42. Masciocchi, N.; Ardizzoia, G. A.; Maspero, A.; La Monica, G.; Sironi, A.; Inorg. Chem. 1999, 38, 3657.

43. Masciocchi, N.; Ardizzoia, G. A.; Brenna, S.; La Monica, G.; Maspero, A.; Galli, S.; Sironi, A.; Inorg. Chem. 2002, 41, 6080.

44. Netto, A. V. G.; Frem, R. C. G.; Mauro, A. E.; Polyhedron 2005, 24, 1086; Netto, A. V. G.; Frem, R. C. G.; Mauro, A. E.; Mol. Cryst. Liq. Cryst. 2002 $374,255$.

45. Casarin, M.; Corvaja, C.; Di Nicola, C.; Falcomer, D.; Franco, L.; Monari, M.; Pandolfo, L.; Pettinari, C.; Piccinelli, F.; Inorg. Chem. 2005, 44, 6265.

46. Cingolani, A.; Galli, S.; Masciocchi, N.; Pandolfo, L.; Pettinari, C.; Sironid, A.; Dalton Trans. 2006, 2479.

47. Masciocchi, N.; Galli, S.; Alberti, E.; Sironi, A.; Di Nicola, C.; Pettinari, C.; Pandolfo, L.; Inorg. Chem. 2006, 45, 9064.

48. Allen, F. H.; Howard, J. A. K.; Hoy, V. J.; Desiraju, G. R.; Reddy, D. S.; Wilson, C. C.; J. Am. Chem. Soc. 1996, 118, 4081.

49. Broder, C. K.; Davidson, M. G.; Forsyth, V. T.; Howard, J. A. K.; Lamb, S.; Mason, S. A.; Cryst. Growth Des. 2002, 2,163.

50. Etter, M. C.; Acc. Chem. Res. 1990, 23, 120; Desiraju, G. R.; Angew. Chem. Int. Ed. 2003, 34, 2311.
51. Lumme, P.; Mutikainen, I.; Lindell, E.; Inorg. Chim. Acta 1983, 71, 217.

52. Sun, Y.-J.; Chen, X.-Y.; Cheng, P.; Yan, S.-P.; Liao, D.-Z.; Jiang, Z.-H.; Shen, P.-W.; J. Mol. Struct. 2002, 613, 167

53. Takahashi, P. M.; Melo, L. P.; Frem, R. C. G.; Netto, A. V. G.; Mauro, A. E.; Santos, R. H. A.; Ferreira, J. G.; J. Mol. Struct. 2006, 783, 161.

54. Zhang, C.; Janiak, C.; Brombacher, C. H.; Z. Naturforsch., B: Chem. Sci. 2001, 56, 1205.

55. Goher, M. A. S.; Mautner, F. A.; Abu-Youssef, M. A. M.; Trans. Met. Chem 1999, 24, 29.

56. Rouhollahi1, A.; Kiaie, F. M.; Ghasemi, J.; Kubista, M.; Polish J. Chem. 2005, 79, 1251.

57. Netto, A. V. G.; Mauro, A. E.; Frem, R. C. G.; de Almeida, E. T.; Santana, A. M.; de Souza Jr., J.; Santos, R. H. A.; Inorg. Chim. Acta 2003, 350, 252 .

58. Nangia, A.; Desiraju, G. R.; Top. Curr. Chem. 1998, 198, 57.

59. Aakeroy, C. B.; Seddon, K. R.; Chem. Soc. Rev. 1993, 22, 397.

60. Segundo Seddon e Aakeroy, ${ }^{59}$ redes estabilizadas por ligações de hidrogênio são codificadas de acordo com a seguinte simbologia: $G^{a}{ }_{d}(n)$, onde $\mathrm{G}=$ designador de padrão, que descreve o tipo de ligação de hidrogênio ( $S$ para ligações intramoleculares, $C$ para cadeias infinitas, $R$ para anéis intermoleculares e $D$ para dímeros acíclicos e outras estruturas finitas); d = número de átomos doadores de ligação de hidrogênio; $\mathrm{a}=$ número de átomos aceitadores de ligação de hidrogênio; $\mathrm{n}=$ número de átomos em um anel.

61. Khripun, A. V.; Selivanov, S. I.; Kukushkin, V. Y.; Haukka, M.; Inorg. Chim. Acta 2006, 359, 320.

62. Sakai, K.; Tomita, Y.; Ue, T.; Goshima, K.; Ohminato, M.; Tsubomura, T.; Matsumoto, K.; Ohmura, K.; Kawakami, K.; Inorg. Chim. Acta 2000, 297, 64.

63. Gimenez, R.; Elduque, A.; Lopez, J. A.; Barbera, J.; Cavero, E.; Lantero, I.; Oro, L. A.; Serrano, J. L.; Inorg. Chem. 2006, 45, 10363; Renard, S. L.; Franken, A.; Kilner, C. A.; Kennedy, J. D.; Halcrow, M. A.; New J. Chem. 2002, 26, 1634

64. Liang, M.; Liao, D.-Z.; Jiang, Z.-H.; Yan, S.-P.; Cheng, P.; Inorg. Chem. Commun. 2004, 7, 173

65. Hong, C. S.; Yoon, J. H.; Lim, J. H.; Ko, H. H.; Eur. J. Inorg. Chem. 2005, 4818 .

66. Ara, I.; Forniés, J.; Lasheras, R.; Martín, A.; Sicilia, V.; Eur. J. Inorg. Chem. 2006, 948.

67. Nishio, M.; Hirota, M.; Umezawa, Y.; The $C-H \cdots \pi$ Interaction. Evidence, Nature and Consequences, Wiley-VCH: New York, 1998; Suezawa, H. Hashimoto, T.; Tsuchinaga, K.; Yoshida, T.; Yuzuri, T.; Sakakibara, K. Hirota, M.; Nishio, M.; J. Chem. Soc., Perkin Trans. 2000, 2, 1243 Suezawa, H.; Yoshida, T.; Hirota, M.; Takahashi, H.; Umezawa, Y.; Honda, K.; Tsuboyama, S.; Nishio, M.; J. Chem. Soc., Perkin Trans. 2001, 2, 2053

68. Silva, P. B.; Frem, R. C. G.; Netto, A. V. G.; Mauro, A. E.; Ferreira, J. G.; Santos, R. H. A.; Inorg. Chem. Commun. 2006, 9, 235.

69. Yilmaz, V. T.; Hamamci, S.; Buyukgungor, O.; Z. Naturforsch., B: Chem. Sci. 2006, 61, 189

70. Singh, U. P.; Sharma, A. K.; Tyagi, P.; Upreti, S.; Singh, R. K.; Polyhedron 2006, $25,3628$.

71. Cram, D. J.; Cram, J. M.; Acc. Chem. Res. 1978, 11, 8.

72. Renard, S. L.; Kilner, C. A.; Fisher, J.; Halcrow, M. A.; Dalton Trans. 2002, 4206; Liu, X.; Kilner, C. A.; Halcrow, M. A.; Chem. Commun. 2002, 704.

73. Renard, S. L.; Sylvestre, I.; Barrett, S. A.; Kilner, C. A.; Halcrow, M. A.; Inorg. Chem. 2006, 45, 8711

74. Nieto, S.; Pérez, J.; Riera, L.; Riera, V.; Miguel, D.; Chem. Eur. J. 2006, $12,2244$.

75. Gallego, M. L.; Ovejero, P.; Cano, M.; Heras, J. V.; Campo, J. A.; Pinilla, E.; Torres, M. R.; Eur. J. Inorg. Chem. 2004, 3089.

76. Torralba, M. C.; Cano, M.; Campo, J. A.; Heras, J. V.; Pinilla, E.; Torres, M. R.; Inorg. Chem. Commun. 2006, 9, 1271; Cano, M.; Heras, J. V.; Gallego, M. L.; Perles, J.; Ruiz-Valero, C.; Pinilla, E.; Torres, M. R.; Helv. Chim. Acta 2003, 86, 3194. 